Tohoku Math. J.

67 (2015), 349-381

\title{
SET-VALUED AND FUZZY STOCHASTIC DIFFERENTIAL EQUATIONS IN M-TYPE 2 BANACH SPACES
}

\author{
MAREK T. MALINOWSKI
}

(Received May 31, 2013, revised May 7, 2014)

\begin{abstract}
In this paper we study set-valued stochastic differential equations in M-type 2 Banach spaces. Their drift terms and diffusion terms are assumed to be set-valued and singlevalued respectively. These coefficients are considered to be random which makes the equations to be truely nonautonomous. Firstly we define set-valued stochastic Lebesgue integral in a Banach space. This integral is a set-valued random variable. We state its properties such as additivity with respect to the interval of integration, continuity as a function of the upper limit of integration, integrable boundedness. The existence and uniqueness of solution to setvalued differential equations in M-type 2 Banach space is obtained by a method of successive approximations. We show that the approximations are uniformly bounded and converge to the unique solution. A distance between $n$th approximation and exact solution is estimated and a continuous dependence of solution with respect to the data of the equation is proved. Finally, we construct a fuzzy stochastic Lebesgue integral in a Banach space and examine fuzzy stochastic differential equations in M-type 2 Banach spaces. We investigate properties like those in set-valued cases. All the results are achieved without assumption on separability of underlying sigma-algebra.
\end{abstract}

1. Introduction. To model evolutionary behavior of dynamical systems governed by random forces stochastic differential equations are used [18, 24, 46, 51]. Their infinite dimensional extension is widely treated in [14], where a comprehensive theory of abstract stochastic differential equations in Hilbert spaces is presented. In [8] stochastic integration in M-type 2 Banach spaces has been introduced. The extensive studies on stochastic integration and stochastic differential equations in Banach spaces can be found e.g. in [8, 9, 10, 52]. Applications of stochastic differential equations range over financial and insurance mathematics, biology, physics, engineering, control theory. However in many situations, owing to vague informations on considered system, states of the system cannot be described by single, precise values. Instead of this, some sets of possible values are available or linguistic variables are used to

2010 Mathematics Subject Classification. Primary 60H20; Secondary 60H05, 28B20, 45R05, 93E03.

Key words and phrases. Set-valued stochastic integral, stochastic integration in Banach spaces, set-valued stochastic differential equation, set-valued stochastic integral equation, existence and uniqueness of solution, fuzzy stochastic integral, fuzzy stochastic differential equation. 
describe a set of values. Such uncertainty is known as contingent uncertainty driven by setvalued mappings, other names are vagueness, imprecision, fuzziness. This is a second source of uncertainty (besides stochastic uncertainty) which is encountered in studies of dynamical systems. Two sources of uncertainty were studied in the subjects of set-valued Markov processes [19, 20], convergence of set-valued random variables [21, 29, 33, 47], definition and properties of fuzzy random variables $[26,45,48,49]$. The stochastic and contingent uncertainties have also been combined in considerations of stochastic differential inclusions (see $[1,2,3,4,6,12,25,27,31,55])$.

In this paper we study somewhat different new tools which can be applied in handling dynamical systems subjected to stochastic and contingent uncertainties. Namely, we are interested in set-valued stochastic differential equations considered in the framework of the martingale type 2 Banach spaces $\mathcal{X}$ (M-type 2 Banach spaces). As distinct from stochastic differential inclusions where solutions are single-valued stochastic processes, solutions to setvalued stochastic differential equations are considered to be set-valued stochastic processes satisfying some measurability conditions and their sample paths are continuous with respect to the Hausdorff metric in the set of nonemtpy closed bounded and convex subsets of the underlying Banach space. Some studies in this direction were proposed in [32] in the setting of the space $\mathbb{R}^{d}$ and in $[41,54]$ in the setting of an M-type 2 Banach space. In these papers some theorems on existence and uniqueness of solution were proven. They were established under assumptions that Lipschitz and linear growth conditions are satisfied by drift and diffusion coefficients of the equation. It was also justified that diffusion coefficient should be single-valued rather than set-valued to consider well possed set-valued stochastic differential equations with solutions being the set-valued stochastic processes. In $[41,54]$ the Banach space-valued stochastic Itô integral has been used, where the integrand is an operator-valued stochastic process and the integrator is an $\mathcal{X}$-valued Wiener process. In this paper, as in [41, 54], we study such equations with set-valued drift and single-valued diffusion. However we allow the coefficients to be random, which makes our equations to be truely nonautonomous. Instead of initial value in the form of set-valued random variable $X_{0}$, we consider an initial set-valued stochastic process $\Phi$. All our results are achieved without assumption on the separability of underlying sigma-algebra $\mathcal{A}$ with respect to the probability measure $P$, whereas such a condition is very important in [41, 54]. The set-valued stochastic Lebesgue integral used here is defined in a different way from that proposed in $[41,54]$. The presented properties of this integral are useful in proving the results concerning set-valued stochastic differential equations, i.e., existence and uniqueness of solution and continuous dependence of solution on data of the equation. 
Instead of linear growth condition we impose a weaker condition of a boundedness type. We further study fuzzy stochastic differential equations in M-type 2 Banach spaces. An initial research in this subject is contained in $[34,35,36,37,38,39,40]$. In this paper in a Banach space setting, we introduce a notion of fuzzy stochastic integral with respect to the Lebesgue measure. It is needed to formulate and study, for the first time, the fuzzy stochastic differential equations in M-type 2 Banach spaces.

The paper is organized as follows: In Section 2 we collect some background material to make the paper self-contained. We recall the definitions and some known properties concerning measurable multifunctions, set-valued stochastic processes, fuzzy sets, measurablity of fuzzy mappings, fuzzy stochastic processes and stochastic integration in M-type 2 Banach spaces with respect to Banach space-valued Wiener process. In Section 3 we define a set-valued stochastic Lebesgue integral in a Banach space and give some of its properties which are used for considering the set-valued stochastic differential equations. We prove, in Section 4, existence and uniqueness of solution to such the equations by a usage of the Picard-type approximations sequence. We estimate the distance between $n$th approximation and exact solution. Also we show that the solution depends continuously on the equation's data. In Section 5 we construct a fuzzy stochastic Lebesgue integral in a Banach space. Then we state some properties of this integral. Finally, in Section 6, we study the fuzzy stochastic differential equations in M-type 2 Banach spaces. The results concerning solutions to such the equations, which are parallel to those of Section 4 , are presented.

2. Preliminaries. Let $(\mathcal{X},\|\cdot\| \mathcal{X})$ denote a separable Banach space (in the paper by $\mathcal{X}$ we will also denote M-type 2 Banach space or separable M-type 2 Banach space thus the meaning of $\mathcal{X}$ will change from time to time, but each time we will state clearly what is meant by $\mathcal{X})$. By $\mathcal{K}_{c}^{b}(\mathcal{X})$ we mean the family of all nonempty, closed, bounded and convex subsets of $\mathcal{X}$. The set $\mathcal{K}_{c}^{b}(\mathcal{X})$ endowed with the Hausdorff metric $H_{\mathcal{X}}$ becomes a complete metric space (cf. $[5,11,23])$. Let us recall that

$$
H_{\mathcal{X}}(A, B):=\max \left\{\sup _{a \in A} \operatorname{dist}_{\mathcal{X}}(a, B), \sup _{b \in B} \operatorname{dist}_{\mathcal{X}}(b, A)\right\}
$$

where $\operatorname{dist}_{\mathcal{X}}(a, B):=\inf _{b \in B}\|a-b\|_{\mathcal{X}}$. In the set $\mathcal{K}_{c}^{b}(\mathcal{X})$ one can define addition and scalar multiplication:

$$
A+B:=\{a+b: a \in A, b \in B\}, \quad r \cdot A:=\{r a: a \in A\}, \quad A, B \in \mathcal{K}_{c}^{b}(\mathcal{X}), \quad r \in \mathbb{R}
$$


Then $\left(\mathcal{K}_{c}^{b}(\mathcal{X}),+, \cdot\right)$ has a semilinear structure. The following property will be used frequently. For nonempty subsets $A_{1}, A_{2}, B_{1}, B_{2}$ of $\mathcal{X}$ it holds

$$
H_{\mathcal{X}}\left(A_{1}+A_{2}, B_{1}+B_{2}\right) \leqslant H_{\mathcal{X}}\left(A_{1}, B_{1}\right)+H_{\mathcal{X}}\left(A_{2}, B_{2}\right) .
$$

Denote $I:=[0, T]$, where $0<T<\infty$. Let $\left(\Omega, \mathcal{A},\left\{\mathcal{A}_{t}\right\}_{t \in I}, P\right)$ be a complete filtered probability space satisfying the usual hypotheses, i.e. $\left\{\mathcal{A}_{t}\right\}_{t \in I}$ is an increasing and right continuous family of sub- $\sigma$-algebras of $\mathcal{A}$ and $\mathcal{A}_{0}$ contains all $P$-null sets.

Let $\mathcal{M}$ be a set of $\mathcal{A}$-measurable mappings $f: \Omega \rightarrow \mathcal{X}$. The set $\mathcal{M}$ is called decomposable if for every $f_{1}, f_{2} \in \mathcal{M}$ and every $A \in \mathcal{A}$ it holds $f_{1} \mathbf{1}_{A}+f_{2} \mathbf{1}_{\Omega \backslash A} \in \mathcal{M}$.

A set-valued mapping (multifunction) $F: \Omega \rightarrow \mathcal{K}_{c}^{b}(\mathcal{X})$ is said to be $\mathcal{A}$-measurable (or measurable, for short, or set-valued random variable) if it satisfies:

$$
\{\omega \in \Omega: F(\omega) \cap O \neq \emptyset\} \in \mathcal{A} \text { for every open set } O \subset \mathcal{X} .
$$

Define $Z_{O}:=\{A$ being nonempty closed subset of $\mathcal{X}: A \cap O \neq \emptyset\}$ for every open subset $O$ of $\mathcal{X}$. Let $\mathcal{C}:=\left\{Z_{O}: O\right.$ is open subset of $\left.\mathcal{X}\right\}$. It is known (see [54]) that multifunction $F:(\Omega, \mathcal{A}) \rightarrow\left(\mathcal{K}_{c}^{b}(\mathcal{X}), \sigma(\mathcal{C})\right)$ is measurable if, and only if, it is $\mathcal{A} \mid \sigma(\mathcal{C})$-measurable, where $\sigma(\mathcal{C})$ is the $\sigma$-algebra generated by $\mathcal{C}$. A measurable multifunction $F: \Omega \rightarrow \mathcal{K}_{c}^{b}(\mathcal{X})$ is said to be $L^{p}$-integrally bounded ( $p \geq 1$ ), if there exists $h \in L^{p}(\Omega, \mathcal{A}, P ; \mathbb{R}$ ) such that $\|a\|_{\mathcal{X}} \leqslant h(\omega)$ for any $a$ and $\omega$ with $a \in F(\omega)$. It is known (see [22]) that $F$ is $L^{p}$-integrally bounded if, and only if, $\omega \mapsto|F(\omega)| \mathcal{X}$ is in $L^{p}(\Omega, \mathcal{A}, P ; \mathbb{R})$. Two measurable and $L^{p}$ integrally bounded multifunctions $F_{1}, F_{2}$ are considered to be identical if $P$-a.e. it holds $F_{1}(\omega)=F_{2}(\omega)$. The set of measurable and $L^{p}$-integrally bounded multifunctions endowed with the metric $\Delta_{p}\left(F_{1}, F_{2}\right):=\mathbb{E}\left(H_{\mathcal{X}}^{p}\left(F_{1}, F_{2}\right)\right)^{1 / p}$ is a complete metric space. For some comprehensive expositions in this subject we refer the reader to [11, 23, 42].

A mapping $F: I \times \Omega \rightarrow \mathcal{K}_{c}^{b}(\mathcal{X})$ is said to be a set-valued stochastic process if $F(t, \cdot): \Omega$ $\rightarrow \mathcal{K}_{c}^{b}(\mathcal{X})$ is a measurable multifunction for every $t \in I$. If $F(t, \cdot)$ is $\mathcal{A}_{t}$-measurable multifunction (for each $t \in I$ ) then $F$ is called $\left\{\mathcal{A}_{t}\right\}$-adapted. A set-valued stochastic process $F$ is said to be measurable if $F(\cdot, \cdot): I \times \Omega \rightarrow \mathcal{K}_{c}^{b}(\mathcal{X})$ is $\beta(I) \otimes \mathcal{A}$-measurable multifunction, where $\beta(I)$ denotes the Borel $\sigma$-algebra of subsets of $I$. If set-valued stochastic process $F$ is measurable and $\left\{\mathcal{A}_{t}\right\}$-adapted then it is called nonanticipating. It is known that $F$ is nonanticipating if, and only if, $F(\cdot, \cdot)$ is measurable with respect to the following $\sigma$-algebra $\mathcal{N}$

$$
\mathcal{N}:=\left\{A \in \beta(I) \otimes \mathcal{A}: A^{t} \in \mathcal{A}_{t} \text { for every } t \in I\right\},
$$

where $A^{t}:=\{\omega \in \Omega:(t, \omega) \in A\}$. A set-valued stochastic process $F: I \times \Omega \rightarrow \mathcal{K}_{c}^{b}(\mathcal{X})$ is said to be $H_{\mathcal{X}}$-continuous if $P$-a.a. its sample paths, i.e. the mappings $F(\cdot, \omega): I \rightarrow \mathcal{K}_{c}^{b}(\mathcal{X})$, 
are continuous with respect to the metric $H_{\mathcal{X}}$. A measurable set-valued stochastic process $F: I \times \Omega \rightarrow \mathcal{K}_{c}^{b}(\mathcal{X})$ is called $L^{p}$-integrally bounded, $p \geq 1$, if the mapping $(t, \omega) \mapsto$ $|F(t, \omega)| \mathcal{X}$ belongs to $L^{p}(I \times \Omega, \beta(I) \otimes \mathcal{A}, \lambda \times P ; \mathbb{R})$, where $\lambda$ denotes the Lebesgue measure on $(I, \beta(I))$. Two measurable and $L^{p}$-integrally bounded processes $F_{1}, F_{2}: I \times \Omega \rightarrow \mathcal{K}_{c}^{b}(\mathcal{X})$ are considered to be identical if $(\lambda \times P)$-a.e. it holds $F_{1}(t, \omega)=F_{2}(t, \omega)$.

A generalization of the notion of set is the notion of fuzzy set [53]. The latter gained popularity and applicability in systems analysis in industrial mathematics $[16,30,44,56]$. A fuzzy set $u$ of the space $\mathcal{X}$ is understood as a mapping $u: \mathcal{X} \rightarrow[0,1]$. In this way, by means of characteristic function, every ordinary subset of $\mathcal{X}$ is a fuzzy set. Let $\mathcal{F}(\mathcal{X})$ denote the set of all the fuzzy sets of the space $\mathcal{X}$.

For $\alpha \in(0,1]$ the set $[u]_{\alpha}:=\{x \in \mathcal{X}: u(x) \geq \alpha\}$ is called $\alpha$-level of $u$, and the set $[u]_{0}:=\operatorname{cl}_{\mathcal{X}}\{x \in \mathcal{X}: u(x)>0\}$ is called the support of $u$. Here $\mathrm{cl}_{\mathcal{X}}$ stands for the closure in $(\mathcal{X},\|\cdot\| \mathcal{X})$.

By $\mathcal{F}_{c}^{b}(\mathcal{X})$ we mean the set of fuzzy sets $u$ for which it holds $[u]_{\alpha} \in \mathcal{K}_{c}^{b}(\mathcal{X})$ for every $\alpha \in[0,1]$. One of the metrics used in $\mathcal{F}_{c}^{b}(\mathcal{X})$ is the uniform Hausdorff metric $D_{\mathcal{X}}$ defined as

$$
D_{\mathcal{X}}(u, v):=\sup _{\alpha \in[0,1]} H_{\mathcal{X}}\left([u]_{\alpha},[v]_{\alpha}\right) \text { for } u, v \in \mathcal{F}_{c}^{b}(\mathcal{X}) .
$$

The following Skorohod metric $D_{\mathcal{X}}^{S}$ is also useful

$$
D_{\mathcal{X}}^{S}(u, v):=\inf _{\lambda \in \Lambda} \max \left\{\sup _{t \in[0,1]}|\lambda(t)-t|, \sup _{t \in[0,1]} H_{\mathcal{X}}\left(x_{u}(t), x_{v}(\lambda(t))\right)\right\},
$$

where $\Lambda$ is the set of strictly increasing continuous functions $\lambda:[0,1] \rightarrow[0,1]$ with $\lambda(0)=$ $0, \lambda(1)=1$, and $x_{u}, x_{v}:[0,1] \rightarrow \mathcal{K}_{c}^{b}(\mathcal{X})$ are the càdlàg representations for fuzzy sets $u, v \in$ $\mathcal{F}_{c}^{b}(\mathcal{X})$ (see [13] for details). The metric space $\left(\mathcal{F}_{c}^{b}(\mathcal{X}), D_{\mathcal{X}}\right)$ is complete and non-separable and the space $\left(\mathcal{F}_{c}^{b}(\mathcal{X}), D_{\mathcal{X}}^{S}\right)$ is a Polish metric space.

The addition $\oplus$ and scalar multiplication $\odot$ in $\mathcal{F}_{c}^{b}(X)$ are defined levelwise, i.e.

$$
[u \oplus v]_{\alpha}=[u]_{\alpha}+[v]_{\alpha}, \quad[\lambda \odot u]_{\alpha}=\lambda \cdot[u]_{\alpha} \text { for } u, v \in \mathcal{F}_{c}^{b}(\mathcal{X}) .
$$

The triple $\left(\mathcal{F}_{c}^{b}(\mathcal{X}), \oplus, \odot\right)$ has semilinear structure and

$$
D_{\mathcal{X}}\left(u_{1} \oplus v_{1}, u_{2} \oplus v_{2}\right) \leqslant D_{\mathcal{X}}\left(u_{1}, u_{2}\right)+D_{\mathcal{X}}\left(v_{1}, v_{2}\right) \text { for } u_{1}, u_{2}, v_{1}, v_{2} \in \mathcal{F}_{c}^{b}(\mathcal{X}) .
$$

A fuzzy mapping $u: \Omega \rightarrow \mathcal{F}_{c}^{b}(\mathcal{X})$ is said to be measurable (or the fuzzy random variable) if $[u]_{\alpha}: \Omega \rightarrow \mathcal{K}_{c}^{b}(\mathcal{X})$ is a measurable multifunction for $\alpha \in[0,1]$, where $[u]_{\alpha}(\omega)=$ $[u(\omega)]_{\alpha}$. It is known (see [13]) that $u: \Omega \rightarrow \mathcal{F}_{c}^{b}(\mathcal{X})$ is measurable if, and only if, $u:(\Omega, \mathcal{A})$ $\rightarrow\left(\mathcal{F}_{c}^{b}(\mathcal{X}), \beta_{D_{\mathcal{X}}^{S}}\right)$ is $\mathcal{A} \mid \beta_{D_{\mathcal{X}}^{S}}$-measurable, where $\beta_{D_{\mathcal{X}}^{S}}$ is the $\sigma$-algebra generated by the 
topology induced by $D_{\mathcal{X}}^{S}$. A measurable mapping $u: \Omega \rightarrow \mathcal{F}_{c}^{b}(\mathcal{X})$ is said to be $L^{p}$-integrally bounded $(p \geq 1)$ if $\omega \mapsto\left|[u(\omega)]_{0}\right|_{\mathcal{X}}$ is in $L^{p}(\Omega, \mathcal{A}, P ; \mathbb{R})$.

Similarly to the set-valued case, $f: I \times \Omega \rightarrow \mathcal{F}_{c}^{b}(\mathcal{X})$ is called a fuzzy stochastic process if $[f]_{\alpha}: I \times \Omega \rightarrow \mathcal{K}_{c}^{b}(\mathcal{X})$ is a set-valued stochastic process for $\alpha \in[0,1]$, where $[f]_{\alpha}(t, \omega)=[f(t, \omega)]_{\alpha}$. Also, a fuzzy stochastic process $f: I \times \Omega \rightarrow \mathcal{F}_{c}^{b}(\mathcal{X})$ is $\left\{\mathcal{A}_{t}\right\}$ adapted (measurable, nonanticipating, respectively) if $[f]_{\alpha}: I \times \Omega \rightarrow \mathcal{K}_{c}^{b}(\mathcal{X})$ is $\left\{\mathcal{A}_{t}\right\}$-adapted (measurable, nonanticipating) for $\alpha \in[0,1]$. A fuzzy stochastic process $f: I \times \Omega \rightarrow \mathcal{F}_{c}^{b}(\mathcal{X})$ is called $D_{\mathcal{X}}$-continuous if its sample paths are continuous with respect to the metric $D_{\mathcal{X}}$. A fuzzy stochastic process $f: I \times \Omega \rightarrow \mathcal{F}_{c}^{b}(\mathcal{X})$ is called $L^{p}$-integrally bounded, $p \geq 1$, if $(t, \omega) \mapsto\left|[f(t, \omega)]_{0}\right|_{\mathcal{X}}$ is in $L^{p}(I \times \Omega, \beta(I) \otimes \mathcal{A}, \lambda \times P ; \mathbb{R})$. Two measurable and $L^{p}$-integrally bounded fuzzy stochastic processes $f_{1}, f_{2}$ are considered to be indentical if $(\lambda \times P)$-a.e. it holds $f_{1}(t, \omega)=f_{2}(t, \omega)$.

In the sequel we recall some foundations of the stochastic integration in Banach spaces $[8,9,14,41,52]$ applied in the single-valued and set-valued stochastic differential equations.

Let $\mathcal{X}$ denote a separable Banach space, $\mathcal{H}$ a separable Hilbert space which is continuously and densely embedded into $\mathcal{X}$, and $\mu$ denote a Gaussian measure on Borel $\sigma$-algebra $\beta(\mathcal{X})$ of subsets of $\mathcal{X}$ such that

$$
\int_{\mathcal{X}} \exp \left\{\sqrt{-1}\left\langle x, x^{*}\right\rangle\right\} \mu(d x)=\exp \left\{-\frac{1}{2}\left\|x^{*}\right\|_{\mathcal{H}}^{2}\right\}, \quad x^{*} \in \mathcal{X}^{*} \subset \mathcal{H} .
$$

The Hilbert space $\mathcal{H}$ is called the reproducing kernel Hilbert space of $(\mathcal{X}, \mu)$, and the triple $(\mathcal{X}, \mathcal{H}, \mu)$ is called the abstract Wiener space (cf. [9, 41]).

Let $B(\mathcal{X} ; \mathcal{X})$ denote the set of all bounded linear operators from $\mathcal{X}$ to $\mathcal{X}$, and let $L^{2}(\mathcal{X} ; \mathcal{X}):=L^{2}(\mathcal{X}, \beta(\mathcal{X}), \mu ; \mathcal{X})$ be the set of all Borel measurable mappings $f:(\mathcal{X}, \beta(\mathcal{X}))$ $\rightarrow(\mathcal{X}, \beta(\mathcal{X}))$ such that the norm

$$
\|f\|_{L^{2}(\mathcal{X} ; \mathcal{X})}:=\left(\int_{\mathcal{X}}\|f(x)\|_{\mathcal{X}}^{2} \mu(d x)\right)^{1 / 2}<\infty .
$$

It is known (see [41]) that $B(\mathcal{X} ; \mathcal{X})$ is a subspace of $L^{2}(\mathcal{X} ; \mathcal{X})$.

Similarly as before, let $(\Omega, \mathcal{A}, P)$ be a complete probability space and let $(\mathcal{X}, \mathcal{H}, \mu)$ be an abstract Wiener space. A continuous stochastic process $W: I \times \Omega \rightarrow \mathcal{X}$ is called the $\mathcal{X}$-valued Wiener process if

(i) $W(0)=0$ a.s.,

(ii) the law of random variable $t^{-1 / 2} W(t)$ equals $\mu$ for every $t \in(0, T]$,

(iii) the random variable $W(t)-W(s)$ is independent of $\sigma\{W(u): u \in[0, s]\}$. 
A Banach space $(\mathcal{X},\|\cdot\| \mathcal{X})$ is called M-type 2 (cf. [9]) if there exists a constant $C_{\mathcal{X}}>0$ such that for every $\mathcal{X}$-valued martingale $\left\{M_{k}\right\}$ it holds

$$
\sup _{k} \mathbb{E}\left\|M_{k}\right\|_{\mathcal{X}}^{2} \leqslant C_{\mathcal{X}} \sum_{k} \mathbb{E}\left\|M_{k}-M_{k-1}\right\|_{\mathcal{X}}^{2}
$$

It is known that every Hilbert space is an M-type 2 Banach space, and the Lebesgue function spaces $L^{p}, p>2$, are M-type 2 Banach spaces which are not Hilbert spaces.

Let $\mathcal{X}$ be a separable M-type 2 Banach space and $(\mathcal{X}, \mathcal{H}, \mu)$ an abstract Wiener space. By $L^{2}(I \times \Omega, \mathcal{N}, \lambda \times P ; B(\mathcal{X} ; \mathcal{X}))$ we denote the set of all nonanticipating operator-valued stochastic processes $f: I \times \Omega \rightarrow B(\mathcal{X} ; \mathcal{X})$ such that $\mathbb{E} \int_{I}\|f\|_{L^{2}(\mathcal{X} ; \mathcal{X})}^{2} d t<\infty$. Then for $f \in L^{2}(I \times \Omega, \mathcal{N}, \lambda \times P ; B(\mathcal{X} ; \mathcal{X}))$ and $t \in T$ one defines (cf. [10, 41]) the stochastic Itô integral of $f$ with respect to $\mathcal{X}$-valued Wiener process denoting this integral as usual, i.e. $\int_{0}^{t} f(s) d W(s)$. It has been proved that

(P1) $\int_{0}^{t} f(s) d W(s)$ is a random variable belonging to $L^{2}\left(\Omega, \mathcal{A}_{t}, P ; \mathcal{X}\right)$ and such that

$$
\mathbb{E} \int_{0}^{t} f(s) d W(s)=\theta \mathcal{X},
$$

(P2) the stochastic process $\mathcal{J}: I \times \Omega \rightarrow \mathcal{X}$ defined by

$$
\mathcal{J}(t, \omega):=\left(\int_{0}^{t} f(s) d W(s)\right)(\omega)
$$

is continuous and nonanticipating, also $\mathcal{J}$ is a martingale,

(P3) for every $t \in I$ it holds

$$
\mathbb{E}\left\|\int_{0}^{t} f(s) d W(s)\right\|_{\mathcal{X}}^{2} \leqslant C_{\mathcal{X}} \mathbb{E} \int_{0}^{t}\|f(s)\|_{L^{2}(\mathcal{X} ; \mathcal{X})}^{2} d s,
$$

(P4) for every $t \in I$ it holds

$$
\mathbb{E} \sup _{u \in[0, t]}\left\|\int_{0}^{u} f(s) d W(s)\right\|_{\mathcal{X}}^{2} \leqslant C_{\mathcal{X}} \mathbb{E} \int_{0}^{t}\|f(s)\|_{L^{2}(\mathcal{X} ; \mathcal{X})}^{2} d s .
$$

3. Set-valued stochastic integral with respect to the Lebesgue measure. Let $\mathcal{X}$ be a separable, reflexive Banach space. Consider a measurable and $L^{1}$-integrally bounded set-valued stochastic process $X: I \times \Omega \rightarrow \mathcal{K}_{c}^{b}(\mathcal{X})$. Then

$$
|X(t, \omega)| \mathcal{X} \leqslant m(t, \omega) \text { for a.a. }(t, \omega) \in I \times \Omega,
$$

where $m: I \times \Omega \rightarrow \mathbb{R}_{+}$is a measurable stochastic process with property $\mathbb{E} \int_{I} m(s) d s<\infty$. By Fubini Theorem there exists a $P$-null set $N_{X}$ such that for $\omega \in \Omega \backslash N_{X}$ the mapping $X(\cdot, \omega): I \rightarrow \mathcal{K}_{c}^{b}(\mathcal{X})$ is $\beta(I)$-measurable and $L^{1}$-integrally bounded. Hence for $\omega \in \Omega \backslash N_{X}$ 
we can define the Aumann integral with respect to the Lebesgue measure (see e.g. [7, 22]) as

$$
\int_{I} X(s, \omega) d s:=\left\{\int_{I} x(s) d s: x \in \mathcal{S}(X(\cdot, \omega))\right\}
$$

where $\mathcal{S}(X(\cdot, \omega))$ is the set of all mappings $x: I \rightarrow \mathcal{X}$ which are measurable and Bochner integrable selections of $X(\cdot, \omega)$. It is known (see [22]) that $\mathcal{S}(X(\cdot, \omega))$ is nonempty, bounded, convex, weakly compact and decomposable subset of $L^{1}(I, \beta(I), \lambda ; \mathcal{X})$.

Since $X(\cdot, \omega)$ is $\mathcal{K}_{c}^{b}(\mathcal{X})$-valued and $L^{1}$-integrally bounded, we obtain (see [22]) that $\int_{I} X(s, \omega) d s \in \mathcal{K}_{c}^{b}(\mathcal{X})$. If we additionally define $\int_{I} X(s, \omega) d s:=\{\theta \mathcal{X}\}$ for $\omega \in N_{X}$ then we get a definition of $\int_{I} X(s, \omega) d s$ for every $\omega \in \Omega$. In the sequel we shall show that

$$
\Omega \ni \omega \mapsto \int_{I} X(s, \omega) d s \in \mathcal{K}_{c}^{b}(\mathcal{X})
$$

is a measurable multifunction. Indeed, denoting by $s$ the support function for $X(t, \omega)$ (see e.g. [23]) and applying Proposition 2.2.39 in [23] we obtain that for every $x^{*} \in \mathcal{X}^{*}$

$$
I \times \Omega \ni(t, \omega) \mapsto s\left(x^{*}, X(t, \omega)\right) \in \mathbb{R}
$$

is a measurable stochastic process. Moreover, it is $L^{1}$-integrally bounded because for every $x^{*} \in \mathcal{X}^{*}$ and every $(t, \omega) \in I \times \Omega$

$$
s\left(x^{*}, X(t, \omega)\right) \leqslant \sup \left\{\left\|x^{*}\right\|_{\mathcal{X}^{*}} \cdot\|x\|_{\mathcal{X}}: x \in X(t, \omega)\right\} \leqslant\left\|x^{*}\right\|_{\mathcal{X}^{*}} \cdot m(t, \omega) .
$$

Hence $\Omega \ni \omega \mapsto \int_{I} s\left(x^{*}, X(s, \omega)\right) d s \in \mathbb{R}$ is $\mathcal{A}$-measurable. Applying Proposition 2.5.2 in [23] we get $\int_{I} s\left(x^{*}, X(s, \cdot)\right) d s=s\left(x^{*}, \int_{I} X(s, \cdot) d s\right)$. Hence we obtain the $\mathcal{A}$-measurability of $s\left(x^{*}, \int_{I} X(s, \cdot) d s\right)$. Now, due to Proposition 2.2.39 in [23] we conclude that $\omega \mapsto \int_{I} X(s, \omega) d s$ is a measurable multifunction.

Definition 3.1. The set-valued stochastic integral over interval $[\tau, t] \subset I$ with respect to Lebesgue measure for measurable and $L^{1}$-integrally bounded set-valued stochastic process $X: I \times \Omega \rightarrow \mathcal{K}_{c}^{b}(\mathcal{X})$ is a measurable multifunction denoted by $\int_{\tau}^{t} X(s, \cdot) d s$ and defined as follows

$$
\int_{\tau}^{t} X(s, \omega) d s:=\int_{I} \mathbf{1}_{[\tau, t]}(s) X(s, \omega) d s \quad \text { if } \omega \in \Omega \backslash N_{X}
$$

and

$$
\int_{\tau}^{t} X(s, \omega) d s:=\left\{\theta_{\mathcal{X}}\right\} \quad \text { if } \omega \in N_{X} .
$$

In a similar way we can define this integral over intervals $[\tau, t),(\tau, t],(\tau, t)$. However, since $\lambda$ is non-atomic, they are all equal to $\int_{\tau}^{t} X(s, \cdot) d s$.

REMARK 3.2. If $Y: I \times \Omega \rightarrow \mathcal{K}_{c}(\mathcal{X})$ is a measurable and $L^{1}$-integrally bounded setvalued stochastic process, where $\mathcal{K}_{c}(\mathcal{X})$ denotes the collection of all nonempty closed and 
convex subsets of $\mathcal{X}$, then we get $Y(t, \omega) \in \mathcal{K}_{c}^{b}(\mathcal{X})$ for a.a. $(t, \omega) \in I \times \Omega$. Therefore for a $\mathcal{K}_{c}(\mathcal{X})$-valued $Y$, without any difficulty, proceeding as above we can define the set-valued stochastic integral with respect to the Lebesgue measure.

Obviously, set-valued stochastic integral with respect to the Lebesgue measure, which is a measurable multifunction, can be well defined for all processes $X: I \times \Omega \rightarrow \mathcal{K}_{c}^{b}(\mathcal{X})$ which are measurable and $L^{p}$-integrally bounded, $p \geq 1$. Moreover, we have the following property.

REMARK 3.3. For a measurable and $L^{p}$-integrally bounded process $X: I \times \Omega \rightarrow$ $\mathcal{K}_{c}^{b}(\mathcal{X})$, similarly to the derivations of measurability of $\omega \mapsto \int_{I} X(s, \omega) d s$ we can prove that the set-valued stochastic process $(t, \omega) \mapsto J(t, \omega)$ is measurable (i.e. $\beta(I) \otimes \mathcal{A}$-measurable), where $J: I \times \Omega \rightarrow \mathcal{K}_{c}^{b}(\mathcal{X})$ is defined as $J(t, \omega):=\int_{0}^{t} X(s, \omega) d s$.

The definition of set-valued stochastic integral with respect to the Lebesgue measure is different from that one in [41, 54]. It is similar to the definition used in [32]. This allows us to achieve some desired properties of this integral without assumption that the $\sigma$-algebra $\mathcal{A}$ is separable with respect to the probability measure $P$. Such condition is crucial in $[41,54]$.

Now we present some useful properties of the set-valued stochastic integral with respect to the Lebesgue measure.

Proposition 3.4. Assume that $X: I \times \Omega \rightarrow \mathcal{K}_{c}^{b}(\mathcal{X})$ is a measurable and $L^{1}$-integrally bounded set-valued stochastic process. Then for every $\omega \in \Omega$ and every $\tau, t, \eta \in$ $I, \tau<t<\eta$, it holds

$$
\int_{\tau}^{t} X(s, \omega) d s+\int_{t}^{\eta} X(s, \omega) d s=\int_{\tau}^{\eta} X(s, \omega) d s .
$$

Proof. The assertion is obvious for $\omega \in N_{X}$. Consider $\omega \in \Omega \backslash N_{X}$. Then for $a \in$ $\int_{\tau}^{t} X(s, \omega) d s+\int_{t}^{\eta} X(s, \omega) d s$ we obtain that there exist $x_{1}, x_{2} \in \mathcal{S}(X(\cdot, \omega))$ such that $a=$ $\int_{\tau}^{t} x_{1}(s) d s+\int_{t}^{\eta} x_{2}(s) d s$. Since $\mathcal{S}(X(\cdot, \omega))$ is decomposable, we get $x \in \mathcal{S}(X(\cdot, \omega))$, where

$$
x(s):=x_{1}(s) \mathbf{1}_{[0, t]}(s)+x_{2}(s) \mathbf{1}_{(t, T]}(s), \quad s \in I .
$$

Observe that $\int_{\tau}^{\eta} x(s) d s=a$. On the other hand $\int_{\tau}^{\eta} x(s) d s \in \int_{\tau}^{\eta} X(s, \omega) d s$. Hence the inclusion $\int_{\tau}^{t} X(s, \omega) d s+\int_{t}^{\eta} X(s, \omega) d s \subset \int_{\tau}^{\eta} X(s, \omega) d s$ follows.

Now, let us take $a \in \int_{\tau}^{\eta} X(s, \omega) d s$. Then there exists a selection $y \in \mathcal{S}(X(\cdot, \omega))$ such that $a=\int_{\tau}^{\eta} y(s) d s$. It is easy to see that $a=\int_{\tau}^{t} y(s) d s+\int_{t}^{\eta} y(s) d s$. Since $\int_{\tau}^{t} y(s) d s \in$ $\int_{\tau}^{t} X(s, \omega) d s$ and $\int_{t}^{\eta} y(s) d s \in \int_{t}^{\eta} X(s, \omega) d s$, we get $a \in \int_{\tau}^{t} X(s, \omega) d s+\int_{t}^{\eta} X(s, \omega) d s$. 
Proposition 3.5. Assume that $X: I \times \Omega \rightarrow \mathcal{K}_{c}^{b}(\mathcal{X})$ is a measurable and $L^{1}$-integrally bounded set-valued stochastic process. Then for every $\omega \in \Omega$ the mapping $I \ni t \mapsto J(t, \omega) \in \mathcal{K}_{c}^{b}(\mathcal{X})$ is $H_{\mathcal{X}}$-continuous.

Proof. For $\omega \in N_{X}$ the continuity of $J(\cdot, \omega)$ is obvious because we have $J(\cdot, \omega) \equiv$ $\{\theta \mathcal{X}\}$. For $\omega \in \Omega \backslash N_{X}$ and $\tau<t$ we can write, using Proposition 3.4,

$$
\begin{aligned}
H_{\mathcal{X}}(J(\tau, \omega), J(t, \omega)) & =H_{\mathcal{X}}\left(\int_{0}^{\tau} X(s, \omega) d s, \int_{0}^{\tau} X(s, \omega) d s+\int_{\tau}^{t} X(s, \omega) d s\right) \\
& \leqslant\left|\int_{\tau}^{t} X(s, \omega) d s\right|_{\mathcal{X}} .
\end{aligned}
$$

Due to Theorem 4.1 in [22] we get

$$
H_{\mathcal{X}}(J(\tau, \omega), J(t, \omega)) \leqslant \int_{\tau}^{t}|X(s, \omega)| \mathcal{X} d s
$$

The last expression converges to zero when $|t-\tau| \rightarrow 0$, because $X(\cdot, \omega)$ is $L^{1}$-integrally bounded. Hence the assertion follows.

Proposition 3.6. Assume that $X, Y: I \times \Omega \rightarrow \mathcal{K}_{c}^{b}(\mathcal{X})$ are measurable and $L^{p}$ integrally bounded, $p \geq 1$. Then $P$-a.e. it holds: for every $\tau<t$

$$
\sup _{u \in[\tau, t]} H_{\mathcal{X}}^{p}\left(\int_{\tau}^{u} X(s, \omega) d s, \int_{\tau}^{u} Y(s, \omega) d s\right) \leqslant(t-\tau)^{p-1} \int_{\tau}^{t} H_{\mathcal{X}}^{p}(X(s, \omega), Y(s, \omega)) d s
$$

and

$$
H_{\mathcal{X}}^{p}\left(\int_{\tau}^{t} X(s, \omega) d s, \int_{\tau}^{t} Y(s, \omega) d s\right) \leqslant(t-\tau)^{p-1} \int_{\tau}^{t} H_{\mathcal{X}}^{p}(X(s, \omega), Y(s, \omega)) d s .
$$

Proof. Consider $\omega \in\left(\Omega \backslash N_{X}\right) \cap\left(\Omega \backslash N_{Y}\right)$. Then the multifunctions $X(\cdot, \omega), Y(\cdot, \omega)$ are $\beta(I)$-measurable and $L_{\beta(I)}^{1}(\lambda)$-integrally bounded. For $\tau<t$, by Theorem 4 .1 in [22] and Jensen's inequality, we have

$$
\begin{aligned}
\sup _{u \in[\tau, t]} H_{\mathcal{X}}^{p}\left(\int_{\tau}^{u} X(s, \omega) d s, \int_{\tau}^{u} Y(s, \omega) d s\right) & \leqslant \sup _{u \in[\tau, t]}\left(\int_{\tau}^{u} H_{\mathcal{X}}(X(s, \omega), Y(s, \omega)) d s\right)^{p} \\
& \leqslant \sup _{u \in[\tau, t]}(u-\tau)^{p-1} \int_{\tau}^{u} H_{\mathcal{X}}^{p}(X(s, \omega), Y(s, \omega)) d s \\
& \leqslant(t-\tau)^{p-1} \int_{\tau}^{t} H_{\mathcal{X}}^{p}(X(s, \omega), Y(s, \omega)) d s .
\end{aligned}
$$

Hence, also the second inequality follows.

COROLlary 3.7. Under assumptions of Proposition 3.6, for every $\tau<t$ it holds

$$
\mathbb{E} \sup _{u \in[\tau, t]} H_{\mathcal{X}}^{p}\left(\int_{\tau}^{u} X(s) d s, \int_{\tau}^{u} Y(s) d s\right) \leqslant(t-\tau)^{p-1} \mathbb{E} \int_{\tau}^{t} H_{\mathcal{X}}^{p}(X(s), Y(s)) d s
$$


and

$$
\mathbb{E} H_{\mathcal{X}}^{p}\left(\int_{\tau}^{t} X(s) d s, \int_{\tau}^{t} Y(s) d s\right) \leqslant(t-\tau)^{p-1} \mathbb{E} \int_{\tau}^{t} H_{\mathcal{X}}^{p}(X(s), Y(s)) d s .
$$

Proposition 3.8. Assume that $X: I \times \Omega \rightarrow \mathcal{K}_{c}^{b}(\mathcal{X})$ is measurable and $L^{p}$-integrally bounded, $p \geq 1$. Then the set-valued stochastic process $J: I \times \Omega \rightarrow \mathcal{K}_{c}^{b}(\mathcal{X})$ is $L^{p}$-integrally bounded.

ProOF. By Remark 3.3, the set-valued stochastic process $J: I \times \Omega \rightarrow \mathcal{K}_{c}^{b}(\mathcal{X})$ is measurable. Note that

$$
\begin{aligned}
\int_{I \times \Omega}|J(t, \omega)|_{\mathcal{X}}^{p} d t P(d \omega) & =\int_{I} \int_{\Omega}\left|\int_{0}^{t} X(s, \omega) d s\right|_{\mathcal{X}}^{p} P(d \omega) d t \\
& \leqslant \int_{I} \int_{\Omega} t^{p-1} \int_{0}^{t}|X(s, \omega)|_{\mathcal{X}}^{p} d s P(d \omega) d t \\
& \leqslant T^{p} \int_{\Omega} \int_{I}|X(s, \omega)|_{\mathcal{X}}^{p} d s P(d \omega) .
\end{aligned}
$$

By the assumptions, the last expression is finite.

REMARK 3.9. If a set-valued stochastic process $X: I \times \Omega \rightarrow \mathcal{K}_{c}^{b}(\mathcal{X})$ is nonanticipating and $L^{p}$-integrally bounded $(p \geq 1)$, then $J: I \times \Omega \rightarrow \mathcal{K}_{c}^{b}(\mathcal{X})$ is nonanticipating and $L^{p}$-integrally bounded.

4. Set-valued stochastic differential equations in M-type 2 Banach spaces. Let $\mathcal{X}$ be a separable, reflexive, M-type 2 Banach space, $(\mathcal{X}, \mathcal{H}, \mu)$ an abstract Wiener space, $W: I \times$ $\Omega \rightarrow \mathcal{X}$ an $\mathcal{X}$-valued Wiener process defined on a complete probability space $(\Omega, \mathcal{A}, P)$ with filtration $\left\{\mathcal{A}_{t}\right\}$ satisfying usual conditions, the law of random variable $t^{-1 / 2} W(t)$ is equal to $\mu$ for $t \in(0, T]$.

In this section we shall consider the set-valued stochastic differential equations, i.e. the relations of the integral form

$$
X(t)=\Phi(t)+\int_{0}^{t} F(s, X(s)) d s+\left\{\int_{0}^{t} G(s, X(s)) d W(s)\right\}, \quad t \in I,
$$

which shall hold $P$-a.e. and where $\Phi: I \times \Omega \rightarrow \mathcal{K}_{c}^{b}(\mathcal{X}), F: I \times \Omega \times \mathcal{K}_{c}^{b}(\mathcal{X}) \rightarrow \mathcal{K}_{c}^{b}(\mathcal{X})$, $G: I \times \Omega \times \mathcal{K}_{c}^{b}(\mathcal{X}) \rightarrow B(\mathcal{X} ; \mathcal{X})$. The first integral is set-valued stochastic integral with respect to the Lebesgue measure (which is different than that used in [41, 54]) and the second integral is single-valued stochastic Itô integral in Banach space $\mathcal{X}$. Note that we consider random coefficients $F, G$. In $[32,41,54]$ they are not random. The initial data include also a set-valued stochastic process $\Phi$ instead of a measurable multifunction. 
Definition 4.1. By a solution to (4.1) we mean a set-valued stochastic process $X: I$ $\times \Omega \rightarrow \mathcal{K}_{c}^{b}(\mathcal{X})$ which is $\left\{\mathcal{A}_{t}\right\}$-adapted, $H_{\mathcal{X}}$-continuous and satisfies (4.1). A solution $X: I \times$ $\Omega \rightarrow \mathcal{K}_{c}^{b}(\mathcal{X})$ is unique if $P$-a.e. it holds: $H_{\mathcal{X}}(X(t), Y(t))=0$ for every $t \in I$, where $Y: I \times \Omega \rightarrow \mathcal{K}_{c}^{b}(\mathcal{X})$ is any solution to (4.1).

In the investigations of the equation (4.1) we assume that the following conditions are satisfied.

(A1) $\Phi: I \times \Omega \rightarrow \mathcal{K}_{c}^{b}(\mathcal{X})$ is a nonanticipating, $H_{\mathcal{X}}$-continuous set-valued stochastic process such that $\mathbb{E} \sup _{t \in I}|\Phi(t)|_{\mathcal{X}}^{2}<\infty$,

(A2) $F: I \times \Omega \times \mathcal{K}_{c}^{b}(\mathcal{X}) \rightarrow \mathcal{K}_{c}^{b}(\mathcal{X})$ is $\mathcal{N} \otimes \sigma(\mathcal{C}) \mid \sigma(\mathcal{C})$-measurable, $G: I \times \Omega \times$ $\mathcal{K}_{c}^{b}(\mathcal{X}) \rightarrow B(\mathcal{X} ; \mathcal{X})$ is $\mathcal{N} \otimes \sigma(\mathcal{C}) \mid \sigma(B(\mathcal{X} ; \mathcal{X}))$-measurable,

(A3) there exists a constant $K>0$ such that $P$-a.e. it holds

$$
\begin{gathered}
\forall t \in I \quad \forall A, B \in \mathcal{K}_{c}^{b}(\mathcal{X}) \quad H_{\mathcal{X}}^{2}(F(t, \omega, A), F(t, \omega, B)) \leqslant K H_{\mathcal{X}}^{2}(A, B), \\
\forall t \in I \forall A, B \in \mathcal{K}_{c}^{b}(\mathcal{X})\|G(t, \omega, A)-G(t, \omega, B)\|_{L^{2}(\mathcal{X} ; \mathcal{X})}^{2} \leqslant K H_{\mathcal{X}}^{2}(A, B),
\end{gathered}
$$

(A4) there exists a constant $M>0$ such that $P$-a.e. it holds

$$
\forall t \in I \quad \max \left\{|F(t, \omega,\{\theta \mathcal{X}\})|_{\mathcal{X}}^{2},\|G(t, \omega,\{\theta \mathcal{X}\})\|_{L^{2}(\mathcal{X} ; \mathcal{X})}^{2}\right\} \leqslant M .
$$

Instead of the linear growth condition, which was assumed in [32, 41, 54], we impose the boundedness condition (A4).

In the derivations of existence of solution to (4.1) we shall use the method of successive approximations. Therefore we define the sequence $\left\{X_{n}\right\}_{n=0}^{\infty}$ of set-valued stochastic processes as follows

$$
X_{0}(t)=\Phi(t), \quad t \in I,
$$

and for $n=1,2, \ldots$

$$
X_{n}(t)=\Phi(t)+\int_{0}^{t} F\left(s, X_{n-1}(s)\right) d s+\left\{\int_{0}^{t} G\left(s, X_{n-1}(s)\right) d W(s)\right\}, \quad t \in I .
$$

Lemma 4.2. Assume that $\Phi, F, G$ satisfy conditions (A1)-(A4). Then every approximation $X_{n}$ is a nonanticipating, $L^{2}$-integrally bounded, $H_{\mathcal{X}}$-continuous set-valued stochastic process.

Proof. Note that $X_{0}: I \times \Omega \rightarrow \mathcal{K}_{c}^{b}(\mathcal{X})$, which is defined as $X_{0}(t)=\Phi(t)$ for $t \in I$, possesses properties described in thesis of the assertion. It is immediate. Assume that $X_{n-1}$ is nonanticipating, $L^{2}$-integrally bounded and $H_{\mathcal{X}}$-continuous. We shall show that $X_{n}$ has these properties, too. 
Due to (A2) the mappings $F\left(\cdot, \cdot, X_{n-1}(\cdot, \cdot)\right): I \times \Omega \rightarrow \mathcal{K}_{c}^{b}(\mathcal{X})$ and $G\left(\cdot, \cdot, X_{n-1}(\cdot, \cdot)\right): I$ $\times \Omega \rightarrow B(\mathcal{X} ; \mathcal{X})$ are the stochastic processes which are $\mathcal{N} \mid \sigma(\mathcal{C})$-measurable and $\mathcal{N} \mid \beta(B(\mathcal{X} ; \mathcal{X}))$-measurable, respectively. Further observe that, by (A3) and (A4), we get

$$
\begin{aligned}
\mathbb{E} \int_{I}\left|F\left(t, X_{n-1}(t)\right)\right|_{\mathcal{X}}^{2} d t & \leqslant 2 \mathbb{E} \int_{I} H_{\mathcal{X}}^{2}\left(F\left(t, X_{n-1}(t)\right), F(t,\{\theta \mathcal{X}\})\right) d t+2 \mathbb{E} \int_{I}\left|F\left(t,\left\{\theta_{\mathcal{X}}\right\}\right)\right|_{\mathcal{X}}^{2} d t \\
& \leqslant 2 K \mathbb{E} \int_{I}\left|X_{n-1}(t)\right|_{\mathcal{X}}^{2} d t+2 M T<\infty
\end{aligned}
$$

Similarly we have

$$
\begin{aligned}
\mathbb{E} \int_{I}\left\|G\left(t, X_{n-1}(t)\right)\right\|_{L^{2}(\mathcal{X} ; \mathcal{X})}^{2} d t \leqslant & 2 \mathbb{E} \int_{I}\left\|G\left(t, X_{n-1}(t)\right)-G\left(t,\left\{\theta_{\mathcal{X}}\right\}\right)\right\|_{L^{2}(\mathcal{X} ; \mathcal{X})}^{2} d t \\
& +2 \mathbb{E} \int_{I}\left\|G\left(t,\left\{\theta_{\mathcal{X}}\right\}\right)\right\|_{L^{2}(\mathcal{X} ; \mathcal{X})}^{2} d t \\
\leqslant & 2 K \mathbb{E} \int_{I}\left|X_{n-1}(t)\right|_{\mathcal{X}}^{2} d t+2 M T<\infty .
\end{aligned}
$$

Hence the integrals in (4.3) are well defined and form some nonanticipating and $L^{2}$-integrally bounded set-valued and $B(\mathcal{X} ; \mathcal{X})$-valued stochastic processes, respectively. Thus $X_{n}$ is nonanticipating and $L^{2}$-integrally bounded. By Proposition 3.5 and property (P2) of stochastic Itô integral in Banach spaces, we infer that $X_{n}$ is $H_{\mathcal{X}}$-continuous.

Lemma 4.3. Under assumptions of Lemma 4.2 , for every $n \in \mathbb{N}$ it holds

$$
\mathbb{E} \sup _{t \in I}\left|X_{n}(t)\right|_{\mathcal{X}}^{2} \leqslant\left(M_{1}+M_{2} T \mathbb{E} \sup _{t \in I}|\Phi(t)|_{\mathcal{X}}^{2}\right) \exp \left\{M_{2} T\right\}
$$

where $M_{1}=3 \mathbb{E} \sup _{t \in I}|\Phi(t)|_{\mathcal{X}}^{2}+6 T M\left(T+C_{\mathcal{X}}\right), M_{2}=6 K\left(T+C_{\mathcal{X}}\right)$.

Proof. For $t \in I$ we have

$$
\begin{aligned}
\mathbb{E} \sup _{s \in[0, t]}\left|X_{n}(s)\right|_{\mathcal{X}}^{2} \leqslant & 3 \mathbb{E} \sup _{s \in I}|\Phi(s)|_{\mathcal{X}}^{2}+3 \mathbb{E} \sup _{s \in[0, t]}\left|\int_{0}^{s} F\left(r, X_{n-1}(r)\right) d r\right|_{\mathcal{X}}^{2} \\
& +3 \mathbb{E} \sup _{s \in[0, t]}\left\|\int_{0}^{s} G\left(r, X_{n-1}(r)\right) d W(r)\right\|_{\mathcal{X}}^{2} .
\end{aligned}
$$

Applying Corollary 3.7, (2.2), (A3) and (A4) we have

$$
\begin{array}{rl}
\underset{\sup _{s \in[0, t]}\left|X_{n}(s)\right|_{\mathcal{X}}^{2} \leqslant}{\mathbb{E}} & 3 \mathbb{E} \sup _{s \in I}|\Phi(s)|_{\mathcal{X}}^{2}+3 t \mathbb{E} \int_{0}^{t}\left|F\left(r, X_{n-1}(r)\right)\right|_{\mathcal{X}}^{2} d r \\
& +3 C_{\mathcal{X}} \mathbb{E} \int_{0}^{t}\left\|G\left(r, X_{n-1}(r)\right)\right\|_{L^{2}(\mathcal{X} ; \mathcal{X})}^{2} \\
\leqslant & 3 \mathbb{E} \sup _{s \in I}|\Phi(s)|_{\mathcal{X}}^{2}+6 t M\left(t+C_{\mathcal{X}}\right) \\
& +6 K\left(t+C_{\mathcal{X}}\right) \mathbb{E} \int_{0}^{t}\left|X_{n-1}(r)\right|_{\mathcal{X}}^{2} d r
\end{array}
$$




$$
\begin{aligned}
\leqslant & 3 \mathbb{E} \sup _{s \in I}|\Phi(s)|_{\mathcal{X}}^{2}+6 T M\left(T+C_{\mathcal{X}}\right) \\
& +6 K\left(T+C_{\mathcal{X}}\right) \int_{0}^{t} \mathbb{E} \sup _{s \in[0, r]}\left|X_{n-1}(s)\right|_{\mathcal{X}}^{2} d r .
\end{aligned}
$$

Hence, for every $k \in \mathbb{N}$ and every $t \in I$

$$
\max _{1 \leqslant n \leqslant k} \mathbb{E} \sup _{s \in[0, t]}\left|X_{n}(s)\right|_{\mathcal{X}}^{2} \leqslant M_{1}+M_{2} \int_{0}^{t} \max _{1 \leqslant n \leqslant k} \mathbb{E} \sup _{s \in[0, r]}\left|X_{n-1}(s)\right|_{\mathcal{X}}^{2} d r
$$

Applying the inequality

$$
\max _{1 \leqslant n \leqslant k} \mathbb{E} \sup _{s \in[0, t]}\left|X_{n-1}(s)\right|_{\mathcal{X}}^{2} \leqslant \mathbb{E} \sup _{s \in I}|\Phi(s)|_{\mathcal{X}}^{2}+\max _{1 \leqslant n \leqslant k} \mathbb{E} \sup _{s \in[0, t]}\left|X_{n}(s)\right|_{\mathcal{X}}^{2}
$$

we arrive at

$$
\max _{1 \leqslant n \leqslant k} \mathbb{E} \sup _{s \in[0, t]}\left|X_{n}(s)\right|_{\mathcal{X}}^{2} \leqslant M_{1}+M_{2} T \mathbb{E} \sup _{s \in I}|\Phi(s)|_{\mathcal{X}}^{2}+M_{2} \int_{0}^{t} \max _{1 \leqslant n \leqslant k} \mathbb{E} \sup _{s \in[0, r]}\left|X_{n}(s)\right|_{\mathcal{X}}^{2} d r .
$$

By Gronwall's inequality we get

$$
\max _{1 \leqslant n \leqslant k} \mathbb{E} \sup _{s \in[0, t]}\left|X_{n}(s)\right|_{\mathcal{X}}^{2} \leqslant\left(M_{1}+M_{2} T \mathbb{E} \sup _{s \in I}|\Phi(s)|_{\mathcal{X}}^{2}\right) e^{M_{2} t} \text { for } t \in I .
$$

Now the assertion follows easily.

We are in a position to formulate the main result of this section.

THEOREM 4.4. Assume that $\Phi, F, G$ satisfy(A1)-(A4). Then the set-valued stochastic differential equation (4.1) possesses a unique solution.

Proof. We will exploite the sequence defined in (4.2) and (4.3). For $t \in I$ we have

$$
\begin{aligned}
\mathbb{E} \sup _{s \in[0, t]} & H_{\mathcal{X}}^{2}\left(X_{1}(s), X_{0}(s)\right) \\
\leqslant & \mathbb{E} \sup _{s \in[0, t]}\left|\int_{0}^{s} F(r, \Phi(r)) d r+\left\{\int_{0}^{s} G(r, \Phi(r)) d W(r)\right\}\right|_{\mathcal{X}}^{2} \\
\leqslant & 2 \mathbb{E} \sup _{s \in[0, t]}\left|\int_{0}^{s} F(r, \Phi(r)) d r\right|_{\mathcal{X}}^{2}+2 \mathbb{E} \sup _{s \in[0, t]}\left\|\int_{0}^{s} G(r, \Phi(r)) d W(r)\right\|_{\mathcal{X}}^{2} \\
\leqslant & 4 \mathbb{E} \sup _{s \in[0, t]} H_{\mathcal{X}}^{2}\left(\int_{0}^{s} F(r, \Phi(r)) d r, \int_{0}^{s} F(r,\{\theta \mathcal{X}\}) d r\right) \\
& +4 \mathbb{E} \sup _{s \in[0, t]}\left|\int_{0}^{s} F(r,\{\theta \mathcal{X}\}) d r\right|_{\mathcal{X}}^{2} \\
& +4 \mathbb{E} \sup _{s \in[0, t]}\left\|\int_{0}^{s} G(r, \Phi(r)) d W(r)-\int_{0}^{s} G(r,\{\theta \mathcal{X}\}) d W(r)\right\|_{\mathcal{X}}^{2} \\
& +4 \mathbb{E} \sup _{s \in[0, t]}\left\|\int_{0}^{s} G(r,\{\theta \mathcal{X}\}) d W(r)\right\|_{\mathcal{X}}^{2} .
\end{aligned}
$$


By Corollary 3.7, (2.2), (A3) and (A4) we get

$$
\begin{aligned}
\mathbb{E} \sup _{s \in[0, t]} & H_{\mathcal{X}}^{2}\left(X_{1}(s), X_{0}(s)\right) \\
\leqslant & 4 t \mathbb{E} \int_{0}^{t} H_{\mathcal{X}}^{2}(F(r, \Phi(r)), F(r,\{\theta \mathcal{X}\})) d r+4 t \mathbb{E} \int_{0}^{t}|F(r,\{\theta \mathcal{X}\})|_{\mathcal{X}}^{2} d r \\
& +4 C_{\mathcal{X}} \mathbb{E} \int_{0}^{t}\left\|G(r, \Phi(r))-G\left(r,\left\{\theta_{\mathcal{X}}\right\}\right)\right\|_{L^{2}(\mathcal{X} ; \mathcal{X})}^{2} d r \\
& +4 C_{\mathcal{X}} \mathbb{E} \int_{0}^{t}\left\|G\left(r,\left\{\theta_{\mathcal{X}}\right\}\right)\right\|_{L^{2}(\mathcal{X} ; \mathcal{X})}^{2} d r \\
\leqslant & L t
\end{aligned}
$$

where $L=4\left(T+C_{\mathcal{X}}\right)\left(M+K \mathbb{E} \sup _{t \in I}|\Phi(t)|_{\mathcal{X}}^{2}\right)$.

Note that for $n \in \mathbb{N}$ and $t \in I$ we obtain

$$
\mathbb{E} \sup _{s \in[0, t]} H_{\mathcal{X}}^{2}\left(X_{n+1}(s), X_{n}(s)\right) \leqslant 2 K\left(T+C_{\mathcal{X}}\right) \int_{0}^{t} \mathbb{E} \sup _{s \in[0, r]} H_{\mathcal{X}}^{2}\left(X_{n}(s), X_{n-1}(s)\right) d r .
$$

Thus

$$
\mathbb{E} \sup _{t \in I} H_{\mathcal{X}}^{2}\left(X_{n}(t), X_{n-1}(t)\right) \leqslant L\left[2 K\left(T+C_{\mathcal{X}}\right)\right]^{n-1} \frac{T^{n}}{n !} .
$$

By Chebyshev inequality we have

$$
P\left(\sup _{t \in I} H_{\mathcal{X}}^{2}\left(X_{n}(t), X_{n-1}(t)\right)>4^{-n}\right) \leqslant L\left[2 K\left(T+C_{\mathcal{X}}\right)\right]^{n-1} \frac{(4 T)^{n}}{n !} .
$$

Now by Borel-Cantelli lemma

$$
P\left(\sup _{t \in I} H_{\mathcal{X}}\left(X_{n}(t), X_{n-1}(t)\right)>2^{-n} \text { infinitely often }\right)=0 .
$$

Hence there exists $\Omega_{c} \in \mathcal{A}$ such that $P\left(\Omega_{c}\right)=1$ and for every $\omega \in \Omega_{c}$ there exists $n_{0}=n_{0}(\omega)$ such that

$$
\sup _{t \in I} H_{\mathcal{X}}\left(X_{n}(t, \omega), X_{n-1}(t, \omega)\right) \leqslant 2^{-n} \text { for } n \geq n_{0} .
$$

This implies that for every $\omega \in \Omega_{c}$ the sequence $\left\{X_{n}(\cdot, \omega)\right\}$ is uniformly convergent to an $H_{\mathcal{X}}$-continuous multifunction $X(\cdot, \omega): I \rightarrow \mathcal{K}_{c}^{b}(\mathcal{X})$. To complete the definition of $X$ to entire $\Omega$, for $\omega \in \Omega \backslash \Omega_{c}$ we can set $X(\cdot, \omega)$ as a freely chosen multifunction from $I$ to $\mathcal{K}_{c}^{b}(\mathcal{X})$. By estimations and convergences proven above we obtain that $X(t, \cdot): \Omega \rightarrow \mathcal{K}_{c}^{b}(\mathcal{X})$ is $\mathcal{A}_{t}$-measurable and $L^{2}$-integrally bounded multifunction. Hence $X(\cdot, \cdot)$ is nonanticipating, $L^{2}$-integrally bounded and $H_{\mathcal{X}}$-continuous set-valued stochastic process.

In the sequel we show that $X$ is a solution to (4.1). Note that for every $t \in I$ we have

$$
\mathbb{E} H_{\mathcal{X}}^{2}\left(X(t), \Phi(t)+\int_{0}^{t} F(s, X(s)) d s+\left\{\int_{0}^{t} G(s, X(s)) d W(s)\right\}\right)
$$




$$
\begin{aligned}
\leqslant & 2 \mathbb{E} H_{\mathcal{X}}^{2}\left(X(t), X_{n}(t)\right) \\
& +4 \mathbb{E} H_{\mathcal{X}}^{2}\left(\int_{0}^{t} F\left(s, X_{n-1}(s)\right) d s, \int_{0}^{t} F(s, X(s)) d s\right) \\
& +4 \mathbb{E}\left\|\int_{0}^{t} G\left(s, X_{n-1}(s)\right) d W(s)-\int_{0}^{t} G(s, X(s)) d W(s)\right\|_{\mathcal{X}}^{2} .
\end{aligned}
$$

By Corollary 3.7, (2.1) and (A3) we obtain

$$
\begin{aligned}
\mathbb{E} H_{\mathcal{X}}^{2}\left(X(t), \Phi(t)+\int_{0}^{t} F(s, X(s)) d s+\left\{\int_{0}^{t} G(s, X(s)) d W(s)\right\}\right) \\
\leqslant 2 \mathbb{E} H_{\mathcal{X}}^{2}\left(X(t), X_{n}(t)\right)+4 T \mathbb{E} \int_{I} H_{\mathcal{X}}^{2}\left(F\left(s, X_{n-1}(s)\right), F(s, X(s))\right) d s \\
\quad+4 C_{\mathcal{X}} \mathbb{E} \int_{I}\left\|G\left(s, X_{n-1}(s)\right)-G(s, X(s))\right\|_{L^{2}(\mathcal{X} ; \mathcal{X})}^{2} d s \\
\leqslant 2 \mathbb{E} H_{\mathcal{X}}^{2}\left(X(t), X_{n}(t)\right)+4\left(T+C_{\mathcal{X}}\right) K \int_{I} \mathbb{E} H_{\mathcal{X}}^{2}\left(X_{n-1}(s), X(s)\right) d s .
\end{aligned}
$$

Since $\mathbb{E} H_{\mathcal{X}}^{2}\left(X_{n}(t), X(t)\right) \stackrel{n \rightarrow \infty}{\longrightarrow} 0$ and $\sup _{n} \mathbb{E} \sup _{t \in I}\left|X_{n}(t)\right|_{\mathcal{X}}^{2}<$ const (due to Lemma 4.3), we infer, by the Lebesgue dominated convergence theorem, that

$$
\int_{I} \mathbb{E} H_{\mathcal{X}}^{2}\left(X_{n-1}(s), X(s)\right) d s \stackrel{n \rightarrow \infty}{\longrightarrow} 0
$$

Hence $\mathbb{E} H_{\mathcal{X}}^{2}\left(X(t), \Phi(t)+\int_{0}^{t} F(s, X(s)) d s+\left\{\int_{0}^{t} G(s, X(s)) d W(s)\right\}\right)=0$ for every $t \in$ $I$. This implies that for every fixed $t \in I$ it holds

$$
H_{\mathcal{X}}\left(X(t), \Phi(t)+\int_{0}^{t} F(s, X(s)) d s+\left\{\int_{0}^{t} G(s, X(s)) d W(s)\right\}\right)=0 \quad P \text {-a.e. }
$$

By $H_{\mathcal{X}}$-continuity of the involved processes we get that $P$-a.e. for every $t \in I$ it holds

$$
H_{\mathcal{X}}\left(X(t), \Phi(t)+\int_{0}^{t} F(s, X(s)) d s+\left\{\int_{0}^{t} G(s, X(s)) d W(s)\right\}\right)=0 .
$$

Finally we prove uniqueness of the solution $X$. Suppose that $Y: I \times \Omega \rightarrow \mathcal{K}_{c}^{b}(\mathcal{X})$ is also a solution to (4.1). Then for every $t \in I$

$$
\begin{aligned}
\mathbb{E} \sup _{s \in[0, t]} H_{\mathcal{X}}^{2}(X(s), Y(s)) \\
\leqslant 2 \mathbb{E} \sup _{s \in[0, t]} H_{\mathcal{X}}^{2}\left(\int_{0}^{s} F(r, X(r)) d r, \int_{0}^{s} F(r, Y(r)) d r\right) \\
\quad+2 \mathbb{E} \sup _{s \in[0, t]}\left\|\int_{0}^{s} G(r, X(r)) d W(r)-\int_{0}^{s} G(r, Y(r)) d W(r)\right\|_{\mathcal{X}}^{2} \\
\leqslant 2 K\left(T+C_{\mathcal{X}}\right) \int_{0}^{t} \mathbb{E} \sup _{s \in[0, r]} H_{\mathcal{X}}^{2}(X(s), Y(s)) d r .
\end{aligned}
$$


Due to Gronwall's inequality we infer that $\mathbb{E} \sup _{s \in[0, t]} H_{\mathcal{X}}^{2}(X(s), Y(s))=0$ for every $t \in I$. This leads us to the conclusion that $P$-a.e. it holds

$$
\sup _{s \in I} H_{\mathcal{X}}(X(s), Y(s))=0
$$

which ends the proof.

PROPOSITION 4.5. Under assumptions of Theorem 4.4 for the solution X to (4.1) and the $n^{\text {th }}$ approximation $X_{n}$ it holds

$$
\mathbb{E} \sup _{t \in I} H_{\mathcal{X}}^{2}\left(X_{n}(t), X(t)\right) \leqslant 2 L T \exp \left\{4 K T\left(T+C_{\mathcal{X}}\right)\right\} \frac{\left[2 K T\left(T+C_{\mathcal{X}}\right)\right]^{n}}{n !},
$$

where $L=4\left(T+C_{\mathcal{X}}\right)\left(M+K \mathbb{E} \sup _{t \in I}|\Phi(t)|_{\mathcal{X}}^{2}\right)$. In particular,

$$
\mathbb{E} \sup _{t \in I} H_{\mathcal{X}}^{2}\left(X_{n}(t), X(t)\right) \rightarrow 0 \text { as } n \rightarrow \infty .
$$

PROOF. Note that for every $t \in I$

$$
\begin{aligned}
\mathbb{E} \sup _{s \in[0, t]} H_{\mathcal{X}}^{2}\left(X_{n}(s), X(s)\right) \leqslant & 2 K\left(T+C_{\mathcal{X}}\right) \mathbb{E} \int_{0}^{t} H_{\mathcal{X}}^{2}\left(X_{n-1}(r), X(r)\right) d r \\
\leqslant & 4 K\left(T+C_{\mathcal{X}}\right) \int_{0}^{t}\left[\mathbb{E} \sup _{s \in[0, r]} H_{\mathcal{X}}^{2}\left(X_{n-1}(s), X_{n}(s)\right)\right. \\
& \left.+\mathbb{E} \sup _{s \in[0, r]} H_{\mathcal{X}}^{2}\left(X_{n}(s), X(s)\right)\right] d r .
\end{aligned}
$$

Therefore, by (4.4),

$$
\begin{aligned}
\mathbb{E} \sup _{s \in[0, t]} H_{\mathcal{X}}^{2}\left(X_{n}(s), X(s)\right) \leqslant & 2 L T \frac{\left[2 K T\left(T+C_{\mathcal{X}}\right)\right]^{n}}{n !} \\
& +4 K\left(T+C_{\mathcal{X}}\right) \int_{0}^{t} \mathbb{E} \sup _{s \in[0, r]} H_{\mathcal{X}}^{2}\left(X_{n}(s), X(s)\right) d r .
\end{aligned}
$$

Hence we arrive at the inequality

$$
\mathbb{E} \sup _{s \in[0, t]} H_{\mathcal{X}}^{2}\left(X_{n}(s), X(s)\right) \leqslant 2 L T \frac{\left[2 K T\left(T+C_{\mathcal{X}}\right)\right]^{n}}{n !} e^{4 K\left(T+C_{\mathcal{X}}\right) t}
$$

for every $t \in I$, from which the assertion follows.

In the sequel we show that the solution to (4.1) depends continuously on the data of the equation. Consider the equation (4.1) and the equation

$$
\tilde{X}(t)=\tilde{\Phi}(t)+\int_{0}^{t} \tilde{F}(s, \tilde{X}(s)) d s+\left\{\int_{0}^{t} \tilde{G}(s, \tilde{X}(s)) d W(s)\right\}, \quad t \in I .
$$


Proposition 4.6. Assume that $\Phi, \tilde{\Phi}, F, \tilde{F}, G, \tilde{G}$ satisfy (A1)-(A4). Then for the solution $X$ to (4.1) and the solution $\tilde{X}$ to (4.5) it holds

$$
\begin{aligned}
& \mathbb{E} \sup _{t \in I} H_{\mathcal{X}}^{2}(\tilde{X}(t), X(t)) \\
& \leqslant {\left[3 \mathbb{E} \sup _{t \in I} H_{\mathcal{X}}^{2}(\tilde{\Phi}(t), \Phi(t))+6 T \mathbb{E} \int_{I} H_{\mathcal{X}}^{2}(\tilde{F}(s, X(s)), F(s, X(s))) d s\right.} \\
&\left.+6 C_{\mathcal{X}} \mathbb{E} \int_{I}\|\tilde{G}(s, X(s))-G(s, X(s))\|_{L^{2}(\mathcal{X} ; \mathcal{X})}^{2} d s\right] \exp \left\{6 K T\left(T+C_{\mathcal{X}}\right)\right\} .
\end{aligned}
$$

Proof. For $t \in I$ we have

$$
\begin{aligned}
& \mathbb{E} \sup _{s \in[0, t]} H_{\mathcal{X}}^{2}(\tilde{X}(s), X(s)) \\
& \leqslant 3 \mathbb{E} \sup _{s \in I} H_{\mathcal{X}}^{2}(\tilde{\Phi}(s), \Phi(s)) \\
&+3 \mathbb{E} \sup _{s \in[0, t]} H_{\mathcal{X}}^{2}\left(\int_{0}^{s} \tilde{F}(r, \tilde{X}(r)) d r, \int_{0}^{s} F(r, X(r)) d r\right) \\
&+3 \mathbb{E} \sup _{s \in[0, t]}\left\|\int_{0}^{s} \tilde{G}(r, \tilde{X}(r)) d W(r)-\int_{0}^{s} G(r, X(r)) d W(r)\right\|_{\mathcal{X}}^{2} \\
& \leqslant 3 \mathbb{E} \sup _{s \in I} H_{\mathcal{X}}^{2}(\tilde{\Phi}(s), \Phi(s))+6 T \mathbb{E} \int_{I} H_{\mathcal{X}}^{2}(\tilde{F}(r, X(r)), F(r, X(r))) d r \\
&+6 C_{\mathcal{X}} \mathbb{E} \int_{I}\|\tilde{G}(r, X(r))-G(r, X(r))\|_{L^{2}(\mathcal{X} ; \mathcal{X})}^{2} d r \\
&+6 K\left(T+C_{\mathcal{X}}\right) \int_{0}^{t} \mathbb{E} \sup _{s \in[0, r]} H_{\mathcal{X}}^{2}(\tilde{X}(s), X(s)) d r .
\end{aligned}
$$

Using Gronwall's inequality we are led to the end of the proof.

Consider the equation (4.1) and the equations

$$
Y_{n}(t)=\Phi_{n}(t)+\int_{0}^{t} F_{n}\left(s, Y_{n}(s)\right) d s+\left\{\int_{0}^{t} G_{n}\left(s, Y_{n}(s)\right) d W(s)\right\}, \quad t \in I,
$$

$n \in \mathbb{N}$.

Corollary 4.7. Assume that $\Phi, \Phi_{n}, F, F_{n}, G, G_{n}$ satisfy (A1)-(A4). Suppose that

$$
\mathbb{E} \sup _{t \in I} H_{\mathcal{X}}^{2}\left(\Phi_{n}(t), \Phi(t)\right) \rightarrow 0 \text { as } n \rightarrow \infty
$$

and for every $A \in \mathcal{K}_{c}^{b}(\mathcal{X})$

$$
\begin{gathered}
\mathbb{E} \int_{I} H_{\mathcal{X}}^{2}\left(F_{n}(s, A), F(s, A)\right) \rightarrow 0 \text { as } n \rightarrow \infty, \\
\mathbb{E} \int_{I}\left\|G_{n}(s, A)-G(s, A)\right\|_{L^{2}(\mathcal{X} ; \mathcal{X})}^{2} \rightarrow 0 \text { as } n \rightarrow \infty .
\end{gathered}
$$


Then for the solution $X$ to (4.1) and the solutions $Y_{n}$ to (4.6) it holds

$$
\mathbb{E} \sup _{t \in I} H_{\mathcal{X}}^{2}\left(Y_{n}(t), X(t)\right) \rightarrow 0 \text { as } n \rightarrow \infty
$$

5. Fuzzy stochastic Lebesgue integral in Banach spaces. In this section we define a notion of a fuzzy stochastic Lebesgue integral and investigate some its properties.

Let $\mathcal{X}$ be a separable, reflexive Banach space. In what follows we shall construct fuzzy stochastic Lebesgue integral. The following known lemmata (see [43]) will be useful.

Lemma 5.1. Let $u \in \mathcal{F}(\mathcal{X})$. Then

(i) $[u]_{\alpha_{1}} \supset[u]_{\alpha_{2}}$ for $0 \leqslant \alpha_{1} \leqslant \alpha_{2} \leqslant 1$,

(ii) $[u]_{\alpha}=\bigcap_{n=1}^{\infty}[u]_{\alpha_{n}}$, where $\left\{\alpha_{n}\right\} \subset[0,1]$ and $\alpha_{n} \nearrow \alpha$.

The converse of this lemma also holds.

Lemma 5.2. Let $M \subset \mathcal{X}$ be a set and let $\left\{C_{\alpha}: \alpha \in[0,1]\right\}$ be a family of subsets of $M$ such that

(i) $C_{0}=M$,

(ii) $C_{\alpha_{1}} \supset C_{\alpha_{2}}$ for $0 \leqslant \alpha_{1} \leqslant \alpha_{2}$,

(iii) $C_{\alpha}=\bigcap_{n=1}^{\infty} C_{\alpha_{n}}$, where $\alpha_{n} \nearrow \alpha$. Then there exists a unique $u \in \mathcal{F}(\mathcal{X})$ such that $[u]_{\alpha}=C_{\alpha}$ for every $\alpha \in[0,1]$. Moreover

$$
u(x)= \begin{cases}\sup \left\{\alpha: x \in C_{\alpha}\right\}, & \text { if } x \in M, \\ 0, & \text { if } x \notin M .\end{cases}
$$

A first aim of this section is to define a fuzzy stochastic Lebesgue integral for fuzzy stochastic processes $x: I \times \Omega \rightarrow \mathcal{F}_{c}^{b}(\mathcal{X})$.

THEOREM 5.3. Let $x: I \times \Omega \rightarrow \mathcal{F}_{c}^{b}(\mathcal{X})$ be a measurable and $L^{1}$-integrally bounded fuzzy stochastic process. Then there exists a unique (up to equality $P$-a.e.) fuzzy random variable $U: \Omega \rightarrow \mathcal{F}_{c}^{b}(\mathcal{X})$ such that for every $\alpha \in[0,1]$

$$
[U(\omega)]_{\alpha}=\int_{I}[x(s, \omega)]_{\alpha} d s, \omega \in \Omega,
$$

where the right-hand side is the set-valued stochastic integral with respect to the Lebesgue measure.

Proof. By Fubini's theorem there exists $N_{x} \in \mathcal{A}$ with $P\left(N_{x}\right)=0$ such that for $\omega \in$ $\Omega \backslash N_{x}$ the mapping $x(\cdot, \omega): I \rightarrow \mathcal{F}_{c}^{b}(\mathcal{X})$ is $\beta(I)$-measurable and $L^{1}$-integrally bounded. Hence for every $\alpha \in[0,1]$ the mapping $[x(\cdot, \omega)]_{\alpha}: I \rightarrow \mathcal{K}_{c}^{b}(\mathcal{X})$ is $\beta(I)$-measurable and $L_{\beta(I)}^{1}(\lambda)$-integrally bounded. Thus for fixed $\omega$, similarly as in Section 3 , we can define the 
set-valued Lebesgue-Aumann integral of $[x(\cdot, \omega)]_{\alpha}$ over $I$, i.e.

$$
\int_{I}[x(s, \omega)]_{\alpha} d s:=\left\{\int_{I} j(s) d s: j \in \mathcal{S}\left([x(\cdot, \omega)]_{\alpha}\right)\right\}
$$

and $\int_{I}[x(s, \omega)]_{\alpha} d s \in \mathcal{K}_{c}^{b}(\mathcal{X})$.

Let $0 \leqslant \alpha_{1} \leqslant \alpha_{2} \leqslant 1$. Then, by Lemma 5.1, $[x(s, \omega)]_{\alpha_{2}} \subset[x(s, \omega)]_{\alpha_{1}}$ for every $s \in I$. Hence $\mathcal{S}\left([x(\cdot, \omega)]_{\alpha_{2}}\right) \subset \mathcal{S}\left([x(\cdot, \omega)]_{\alpha_{1}}\right)$ which implies that

$$
\int_{I}[x(s, \omega)]_{\alpha_{2}} d s \subset \int_{I}[x(s, \omega)]_{\alpha_{1}} d s .
$$

Let $\left\{\alpha_{n}\right\} \subset[0,1]$ and $\alpha_{n} \nearrow \alpha$. Then $\mathcal{S}\left([x(\cdot, \omega)]_{\alpha_{1}}\right) \supset \mathcal{S}\left([x(\cdot, \omega)]_{\alpha_{2}}\right) \supset \cdots \supset \mathcal{S}\left([x(\cdot, \omega)]_{\alpha}\right)$. Hence $\mathcal{S}\left([x(\cdot, \omega)]_{\alpha}\right) \subset \bigcap_{n=1}^{\infty} \mathcal{S}\left([x(\cdot, \omega)]_{\alpha_{n}}\right)$.

Let $j(\cdot) \in \bigcap_{n=1}^{\infty} \mathcal{S}\left([x(\cdot, \omega)]_{\alpha_{n}}\right)$. It means that for every $n \in \mathbb{N}$ it holds $j(s) \in[x(s, \omega)]_{\alpha_{n}}$ $\lambda$-a.e. Hence $j(s) \in \bigcap_{n=1}^{\infty}[x(s, \omega)]_{\alpha_{n}} \lambda$-a.e. Since $j$ is integrable and $\lambda$-a.e. it holds $j(s) \in$ $[x(s, \omega)]_{\alpha}$, we get $j(\cdot) \in S\left([x(\cdot, \omega)]_{\alpha}\right)$. Therefore we obtain

$$
\mathcal{S}\left([x(\cdot, \omega)]_{\alpha}\right)=\bigcap_{n=1}^{\infty} \mathcal{S}\left([x(\cdot, \omega)]_{\alpha_{n}}\right) .
$$

In the sequel we shall show that

$$
\int_{I}[x(s, \omega)]_{\alpha} d s=\bigcap_{n=1}^{\infty} \int_{I}[x(s, \omega)]_{\alpha_{n}} d s .
$$

Let $a \in \int_{I}[x(s, \omega)]_{\alpha} d s$. Then there exists $j(\cdot) \in \mathcal{S}\left([x(\cdot, \omega)]_{\alpha}\right)$ such that $a=\int_{I} j(s) d s$. On the other hand, by (5.1), for every $n \in \mathbb{N}$ we have $j(\cdot) \in \mathcal{S}\left([x(\cdot, \omega)]_{\alpha_{n}}\right)$. Hence

$$
a \in \bigcap_{n=1}^{\infty}\left\{\int_{I} i(s) d s: i(\cdot) \in \mathcal{S}\left([x(\cdot, \omega)]_{\alpha_{n}}\right)\right\},
$$

which means that $\int_{I}[x(s, \omega)]_{\alpha} d s \subset \bigcap_{n=1}^{\infty} \int_{I}[x(s, \omega)]_{\alpha_{n}} d s$.

Let $b \in \bigcap_{n=1}^{\infty} \int_{I}[x(s, \omega)]_{\alpha_{n}} d s$. Then for every $n \in \mathbb{N}$ there exists $j_{n}(\cdot) \in \mathcal{S}\left([x(\cdot, \omega)]_{\alpha_{n}}\right)$ such that $b=\int_{I} j_{n}(s) d s$. Obviously, $\left\{j_{n}(\cdot)\right\}_{n=1}^{\infty} \subset \mathcal{S}\left([x(\cdot, \omega)]_{\alpha_{1}}\right)$. Since $\mathcal{S}\left([x(\cdot, \omega)]_{\alpha_{1}}\right)$ is a weakly compact subset of $L^{1}(I, \beta(I), \lambda ; \mathcal{X})$ then there exist $\left\{j_{n_{k}}(\cdot)\right\}_{k=1}^{\infty}$ and $j(\cdot) \in$ $\mathcal{S}\left([x(\cdot, \omega)]_{\alpha_{1}}\right)$ such that

$$
j_{n_{k}}(\cdot) \stackrel{k \rightarrow \infty}{\longrightarrow} j(\cdot) \text { in the weak topology of } L^{1}(I, \beta(I), \lambda ; \mathcal{X}) .
$$

Since the linear operator $V: L^{1}(I, \beta(I), \lambda ; \mathcal{X}) \rightarrow \mathcal{X}$, defined as $V(f(\cdot)):=\int_{I} f(s) d s$, is norm-to-norm continuous, by Theorem 5.3.15 in [17] we obtain

$$
V\left(j_{n_{k}}(\cdot)\right) \stackrel{k \rightarrow \infty}{\longrightarrow} V(j(\cdot)) \text { in the weak topology of } \mathcal{X} .
$$


We have $V\left(j_{n_{k}}(\cdot)\right)=b$ for every $k \in \mathbb{N}$. Hence $\int_{I} j(s) d s=b$. It remains to show that $j(\cdot) \in$ $\mathcal{S}\left([x(\cdot, \omega)]_{\alpha}\right)$. Let $\ell \in \mathbb{N}$. Note that $\left\{j_{n_{k}}(\cdot), k \geq \ell\right\} \subset \mathcal{S}\left([x(\cdot, \omega)]_{\alpha_{n_{\ell}}}\right)$ and $\mathcal{S}\left([x(\cdot, \omega)]_{\alpha_{n_{\ell}}}\right)$ is weakly closed. Hence $j(\cdot) \in \mathcal{S}\left([x(\cdot, \omega)]_{\alpha_{n_{\ell}}}\right.$ ) for every $\ell \in \mathbb{N}$. Due to (5.1) we obtain $j(\cdot) \in \mathcal{S}\left([x(\cdot, \omega)]_{\alpha}\right)$.

Now, applying Lemma 5.2 we can state that for every $\omega \in \Omega \backslash N_{x}$ there exists a unique fuzzy set $U(\omega)$ in $\mathcal{F}_{c}^{b}(\mathcal{X})$ such that

$$
[U(\omega)]_{\alpha}=\int_{I}[x(s, \omega)]_{\alpha} d s .
$$

For $\omega \in N_{x}$ let us set $U(\omega)=\langle\langle\theta \mathcal{X}\rangle\rangle$, where $\langle\langle a\rangle\rangle$ is the characteristic function of singleton $\{a\} \subset \mathcal{X}$. In this way we defined mapping $U: \Omega \rightarrow \mathcal{F}_{c}^{b}(\mathcal{X})$.

Remembering that $x: I \times \Omega \rightarrow \mathcal{F}_{c}^{b}(\mathcal{X})$ is a measurable and $L^{1}$-integrally bounded fuzzy stochastic process, we obtain that for every $\alpha \in[0,1]$ the set-valued stochastic process $[x]_{\alpha}: I \times \Omega \rightarrow \mathcal{K}_{c}^{b}(\mathcal{X})$ is measurable and $L^{1}$-integrally bounded. Hence $\omega \mapsto \int_{I}[x(s, \omega)]_{\alpha} d s$ $\in \mathcal{K}_{c}^{b}(\mathcal{X})$ is a measurable multifunction for every $\alpha \in[0,1]$. This means that

$$
\Omega \ni \omega \mapsto U(\omega) \in \mathcal{F}_{c}^{b}(\mathcal{X})
$$

is a measurable fuzzy mapping.

Definition 5.4. The measurable fuzzy mapping $U: \Omega \rightarrow \mathcal{F}_{c}^{b}(\mathcal{X})$ described in Theorem 5.3 is called the fuzzy stochastic Lebesgue integral (over interval $I$ ) of the measurable and $L^{1}$-integrally bounded fuzzy stochastic process $x: I \times \Omega \rightarrow \mathcal{F}_{c}^{b}(\mathcal{X})$. This integral will be denoted by $\int_{I} x(s, \cdot) d s$ or just by the symbol $\int_{I} x(s) d s$.

Of course we can consider the integrals of the form

$$
\int_{G} x(s, \cdot) d s:=\int_{I} \mathbf{1}_{G}(s) x(s, \cdot) d s
$$

where $G$ is a measurable subset of $I$. In particular, for $\tau, t \in I, \tau<t$, we have $\int_{\tau}^{t} x(s, \cdot) d s:=$ $\int_{I} \mathbf{1}_{[\tau, t]}(s) x(s, \cdot) d s$. Obviously, the fuzzy stochastic Lebesgue integrals $\int_{I} \mathbf{1}_{(\tau, t]}(s) x(s, \cdot) d s$, $\int_{I} \mathbf{1}_{[\tau, t)}(s) x(s, \cdot) d s, \int_{I} \mathbf{1}_{(\tau, t)}(s) x(s, \cdot) d s$ they are all equal to $\int_{\tau}^{t} x(s, \cdot) d s$.

REMARK 5.5. Let $y: I \times \Omega \rightarrow \mathcal{F}_{c}(\mathcal{X})$ be a measurable and $L^{1}$-integrally bounded fuzzy stochastic process, where $\mathcal{F}_{c}(\mathcal{X})$ denotes the collection of all fuzzy sets $u \in \mathcal{F}(\mathcal{X})$ such that $[u]_{\alpha} \in \mathcal{K}_{c}(\mathcal{X})$ for every $\alpha \in[0,1]$. Then we obtain $y(t, \omega) \in \mathcal{F}_{c}^{b}(\mathcal{X})$ for a.a. $(t, \omega) \in I \times \Omega$ and similarly as above we can define the fuzzy stochastic Lebesgue integral of the $\mathcal{F}_{c}(\mathcal{X})$-valued $y$. 
REMARK 5.6. Let $x: I \times \Omega \rightarrow \mathcal{F}_{c}^{b}(\mathcal{X})$ be measurable and $L^{p}$-integrally bounded, $p \geq 1$. Then the fuzzy stochastic process

$$
I \times \Omega \ni(t, \omega) \mapsto \int_{0}^{t} x(s, \omega) d s \in \mathcal{F}_{c}^{b}(\mathcal{X})
$$

is measurable.

REMARK 5.7. If $x: I \times \Omega \rightarrow \mathcal{F}_{c}^{b}(\mathcal{X})$ is nonanticipating and $L^{p}$-integrally bounded $(p \geq 1)$, then $I \times \Omega \ni(t, \omega) \mapsto \int_{0}^{t} x(s, \omega) d s \in \mathcal{F}_{c}^{b}(\mathcal{X})$ is nonanticipating and $L^{p}$-integrally bounded.

Some properties of the fuzzy stochastic Lebesgue integral will be stated below. We omit their proofs, because they are modifications of the derivations presented in Section 3 for setvalued cases.

Proposition 5.8. Assume that $x: I \times \Omega \rightarrow \mathcal{F}_{c}^{b}(\mathcal{X})$ is a measurable and $L^{1}$-integrally bounded fuzzy stochastic process. Then it holds

$$
\int_{\tau}^{t} x(s, \omega) d s \oplus \int_{t}^{\eta} x(s, \omega) d s=\int_{\tau}^{\eta} x(s, \omega) d s
$$

for every $\omega \in \Omega$ and every $\tau, t, \eta \in I, \tau<t<\eta$.

Proposition 5.9. Assume that $x: I \times \Omega \rightarrow \mathcal{F}_{c}^{b}(\mathcal{X})$ is a measurable and $L^{1}$-integrally bounded fuzzy stochastic process. Then for every $\omega \in \Omega$ the mapping $I \ni t \mapsto \int_{0}^{t} x(s, \omega) d s \in$ $\mathcal{F}_{c}^{b}(\mathcal{X})$ is D $D_{\text {-continuous. }}$

Proposition 5.10. Assume that $x: I \times \Omega \rightarrow \mathcal{F}_{c}^{b}(\mathcal{X})$ is measurable and $L^{p}$-integrally bounded, $p \geq 1$. Then the fuzzy stochastic process $I \times \Omega \ni(t, \omega) \mapsto \int_{0}^{t} x(s, \omega) d s \in \mathcal{F}_{c}^{b}(\mathcal{X})$ is $L^{p}$-integrally bounded.

In the next properties concerning the fuzzy stochastic Lebesgue integral and the examinations of fuzzy stochastic differential equations, it is important to guarantee that the mapping $\Omega \ni \omega \mapsto D_{\mathcal{X}}(u(\omega), v(\omega)) \in \mathbb{R}$ is a random variable, where $u, v$ are the fuzzy random variables. We observe that for the measurability of the mapping $\omega \mapsto D_{\mathcal{X}}(u(\omega), v(\omega))$, the stochastic process $[0,1] \times \Omega \ni(\alpha, \omega) \mapsto H_{\mathcal{X}}\left([u(\omega)]_{\alpha},[v(\omega)]_{\alpha}\right)$ should be separable. In such a case the mapping $\omega \mapsto \sup _{\alpha \in[0,1]} H_{\mathcal{X}}\left([u(\omega)]_{\alpha},[v(\omega)]_{\alpha}\right)$ will be a random variable. This property is not obvious for the fuzzy random variables $u, v$ taking on values from $\mathcal{F}_{c}^{b}(\mathcal{X})$. Therefore we must consider the following class of fuzzy sets

$$
\begin{aligned}
\mathcal{F}_{c}^{b}(\mathcal{X}):= & \left\{u \in \mathcal{F}_{c}^{b}(\mathcal{X}): \alpha \mapsto[u]_{\alpha}\right. \text { is left continuous with respect } \\
& \text { to the Hausdorff metric } \left.H_{\mathcal{X}}\right\} .
\end{aligned}
$$


Notice that the set $\mathcal{F L}_{c}^{b}(\mathcal{X})$ is nonempty. The class $\mathcal{K}_{c}^{b}(\mathcal{X})$ can be embedded into $\mathcal{F}_{c}^{b}(\mathcal{X})$. Also the class of fuzzy sets which have nonempty, compact and convex $\alpha$-cuts form a subset of $\mathcal{F L}_{c}^{b}(\mathcal{X})$. This is indicated below. Denote

$$
\begin{aligned}
\mathcal{F C}(\mathcal{X}):= & \left\{u \in \mathcal{F}_{c}^{b}(\mathcal{X}):[u]_{\alpha}\right. \text { are nonempty, compact } \\
& \text { and convex subsets of } \mathcal{X}, \alpha \in[0,1]\} .
\end{aligned}
$$

\section{LEMMA 5.11. It holds}

$$
\mathcal{F C}(\mathcal{X}) \subset \mathcal{F L}_{c}^{b}(\mathcal{X})
$$

PROOF. It is enough to show that for $u \in \mathcal{F C}(\mathcal{X})$ the mapping $\alpha \mapsto[u]_{\alpha}$ is left continuous with respect to the Hausdorff metric $H_{\mathcal{X}}$. Let $\alpha \in(0,1]$ and $\left\{\alpha_{k}\right\} \subset[0,1]$ be such that $\alpha_{k} \nearrow \alpha$. By Lemma 5.1 for $u \in \mathcal{F C}(\mathcal{X})$

$$
[u]_{\alpha_{1}} \supset[u]_{\alpha_{2}} \supset[u]_{\alpha_{3}} \supset \cdots .
$$

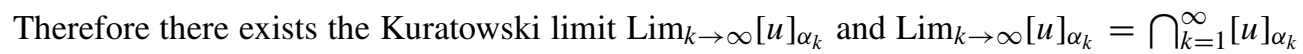
(see [28], page 339). On the other hand, by Lemma 5.1, $\bigcap_{k=1}^{\infty}[u]_{\alpha_{k}}=[u]_{\alpha}$. Hence

$$
\operatorname{Lim}_{k \rightarrow \infty}[u]_{\alpha_{k}}=[u]_{\alpha} .
$$

Now, by Proposition 1.19 in Chap. 7 of [23], we infer that

$$
H_{\mathcal{X}}\left([u]_{\alpha_{k}},[u]_{\alpha}\right) \longrightarrow 0 \text { as } \alpha_{k} \nearrow \alpha
$$

which means that $\alpha \mapsto[u]_{\alpha}$ is left continuous.

Notice that for $u, v \in \mathcal{F}_{c}^{b}(\mathcal{X})$ and $\left\{\alpha_{k}\right\} \subset[0,1], \alpha \in(0,1]$ being such that $\alpha_{k} \nearrow \alpha$ we have

$$
H_{\mathcal{X}}\left([u \oplus v]_{\alpha_{k}},[u \oplus v]_{\alpha}\right) \leqslant H_{\mathcal{X}}\left([u]_{\alpha_{k}},[u]_{\alpha}\right)+H_{\mathcal{X}}\left([v]_{\alpha_{k}},[v]_{\alpha}\right) \longrightarrow 0 \text { as } k \rightarrow \infty .
$$

Hence we obtain the following property.

REMARK 5.12. The set $\mathcal{F}_{c}^{b}(\mathcal{X})$ is closed under operation $\oplus$.

LEMMA 5.13. The metric space $\left(\mathcal{F L}_{c}^{b}(\mathcal{X}), D_{\mathcal{X}}\right)$ is a complete metric space.

Proof. As we mentioned in Preliminaries, $\left(\mathcal{F}_{c}^{b}(\mathcal{X}), D_{\mathcal{X}}\right)$ is a complete metric space. We will show that $\mathcal{F}_{c}^{b}(\mathcal{X})$ is a closed subset in $\left(\mathcal{F}_{c}^{b}(\mathcal{X}), D_{\mathcal{X}}\right)$. Let $\left\{u_{n}\right\}_{n=1}^{\infty} \subset \mathcal{F L}_{c}^{b}(\mathcal{X})$ be the sequence that converges to $u \in \mathcal{F}_{c}^{b}(\mathcal{X})$ in the metric $D_{\mathcal{X}}$. Let $\left\{\alpha_{k}\right\}_{k=1}^{\infty} \subset[0,1], \alpha \in(0,1]$ be such that $\alpha_{k} \nearrow \alpha$. Since

$$
H_{\mathcal{X}}\left([u]_{\alpha_{k}},[u]_{\alpha}\right) \leqslant H_{\mathcal{X}}\left([u]_{\alpha_{k}},\left[u_{n}\right]_{\alpha_{k}}\right)+H_{\mathcal{X}}\left(\left[u_{n}\right]_{\alpha_{k}},\left[u_{n}\right]_{\alpha}\right)+H_{\mathcal{X}}\left(\left[u_{n}\right]_{\alpha},[u]_{\alpha}\right),
$$


we obtain $H_{\mathcal{X}}\left([u]_{\alpha_{k}},[u]_{\alpha}\right) \rightarrow 0$ as $k \rightarrow \infty$. Therefore $u \in \mathcal{F}_{c}^{b}(\mathcal{X})$. Hence closedness of $\mathcal{F}_{c}^{b}$ implies completness of the metric space $\left(\mathcal{F L}_{c}^{b}(\mathcal{X}), D_{\mathcal{X}}\right)$.

For the fuzzy random variables $u, v: \Omega \rightarrow \mathcal{F}_{c}^{b}(\mathcal{X})$ it is possible to obtain that $\omega \mapsto$ $D_{\mathcal{X}}(u(\omega), v(\omega))$ is a random variable. We show this in a collection of lemmata below.

Lemma 5.14. Let $u, v \in \mathcal{F}_{c}^{b}(\mathcal{X})$. Then the mapping $\alpha \mapsto H_{\mathcal{X}}\left([u]_{\alpha},[v]_{\alpha}\right)$ is left continuous.

PROOF. Due to the Rådström theorem [50] the family of nonempty, closed, bounded and convex subsets of $\mathcal{X}$ can be embedded isometrically by an embedding $j$ into a corresponding real normed linear space $E$. We have

$\left\|j\left([u]_{\alpha_{k}}\right)-j\left([u]_{\alpha}\right)-\left(j\left([v]_{\alpha_{k}}\right)-j\left([v]_{\alpha}\right)\right)\right\|_{E} \leqslant\left\|j\left([u]_{\alpha_{k}}\right)-j\left([u]_{\alpha}\right)\right\|_{E}+\left\|j\left([v]_{\alpha_{k}}\right)-j\left([v]_{\alpha}\right)\right\|_{E}$.

Since the right-hand side of the inequality above equals $H_{\mathcal{X}}\left([u]_{\alpha_{k}},[u]_{\alpha}\right)+H_{\mathcal{X}}\left([v]_{\alpha_{k}},[v]_{\alpha}\right)$ and $u, v \in \mathcal{F L}_{c}^{b}(\mathcal{X})$, we get

$$
j\left([u]_{\alpha_{k}}\right)-j\left([v]_{\alpha_{k}}\right) \longrightarrow j\left([u]_{\alpha}\right)-j\left([v]_{\alpha}\right) \text { strongly in } E, \text { as } \alpha_{k} \nearrow \alpha .
$$

This implies that $\left\|j\left([u]_{\alpha_{k}}\right)-j\left([v]_{\alpha_{k}}\right)\right\|_{E} \longrightarrow\left\|j\left([u]_{\alpha}\right)-j\left([v]_{\alpha}\right)\right\|_{E}$ as $\alpha_{k} \nearrow \alpha$. Consequently

$$
H_{\mathcal{X}}\left([u]_{\alpha_{k}},[v]_{\alpha_{k}}\right) \longrightarrow H_{\mathcal{X}}\left([u]_{\alpha},[v]_{\alpha}\right) \text { as } \alpha_{k} \nearrow \alpha,
$$

which ends the proof.

LEMMA 5.15. Let $u, v: \Omega \rightarrow \mathcal{F}_{c}^{b}(\mathcal{X})$ be the fuzzy random variables. Then for every $\alpha \in[0,1]$ the mapping $\omega \mapsto H_{\mathcal{X}}\left([u(\omega)]_{\alpha},[v(\omega)]_{\alpha}\right)$ is a random variable.

PROOF. By definition of the fuzzy random variable, for fixed $\alpha \in[0,1]$ the mappings $\omega \mapsto[u(\omega)]_{\alpha}$ and $\omega \mapsto[v(\omega)]_{\alpha}$ are the set-valued random variables. Hence each one of these set-valued random variables has the Castaing representation, i.e. for every $\omega \in \Omega$

$$
[u(\omega)]_{\alpha}=\operatorname{cl}_{\mathcal{X}}\left\{f_{n, \alpha}(\omega): n \in \mathbb{N}\right\}, \quad[v(\omega)]_{\alpha}=\operatorname{cl}_{\mathcal{X}}\left\{g_{n, \alpha}(\omega): n \in \mathbb{N}\right\},
$$

where $f_{n, \alpha}, g_{n, \alpha}$ for $n \in \mathbb{N}$ are some random variables and selections of $[u]_{\alpha}$ and $[v]_{\alpha}$, respectively. Hence for every $\omega \in \Omega$

$$
\begin{aligned}
& H_{\mathcal{X}}\left([u(\omega)]_{\alpha},[v(\omega)]_{\alpha}\right) \\
& \quad=\max \left\{\sup _{k \in \mathbb{N}} \inf _{\ell \in \mathbb{N}}\left\|f_{k, \alpha}(\omega)-g_{\ell, \alpha}(\omega)\right\| \mathcal{X}, \sup _{\ell \in \mathbb{N}} \inf _{k \in \mathbb{N}}\left\|f_{k, \alpha}(\omega)-g_{\ell, \alpha}(\omega)\right\|_{\mathcal{X}}\right\} .
\end{aligned}
$$

Notice that the right-hand side above represents a random variable, so is the mapping $\omega \mapsto$ $H_{\mathcal{X}}\left([u(\omega)]_{\alpha},[v(\omega)]_{\alpha}\right)$. 
Lemma 5.16. Let $u, v: \Omega \rightarrow \mathcal{F}_{c}^{b}(\mathcal{X})$ be the fuzzy random variables. Then the mapping $\omega \mapsto D_{\mathcal{X}}(u(\omega), v(\omega))$ is a random variable.

Proof. By Lemmata 5.14 and 5.15 the mapping $(\alpha, \omega) \mapsto H_{\mathcal{X}}\left([u(\omega)]_{\alpha},[v(\omega)]_{\alpha}\right)$ is a stochastic process with left continuous sample paths. Therefore it is a separable stochastic process (see [15]). Let $S$ denote a separant (a countable set) for this process. Then $\sup _{\alpha \in[0,1]} H_{\mathcal{X}}\left([u(\omega)]_{\alpha},[v(\omega)]_{\alpha}\right)=\sup _{\alpha \in S} H_{\mathcal{X}}\left([u(\omega)]_{\alpha},[v(\omega)]_{\alpha}\right)$. By Lemma 5.15 the righthand side represents a random variable, so is $\omega \mapsto \sup _{\alpha \in[0,1]} H_{\mathcal{X}}\left([u(\omega)]_{\alpha},[v(\omega)]_{\alpha}\right)$. Since $\sup _{\alpha \in[0,1]} H_{\mathcal{X}}\left([u(\omega)]_{\alpha},[v(\omega)]_{\alpha}\right)=D_{\mathcal{X}}(u(\omega), v(\omega))$, the proof is completed.

COROLlary 5.17. For the fuzzy random variables $u, v: \Omega \rightarrow \mathcal{F C}(\mathcal{X})$, the mapping $\omega \mapsto D_{\mathcal{X}}(u(\omega), v(\omega))$ is a random variable.

Now we proceed with some properties of the fuzzy stochastic Lebesgue integral which are useful in the investigations of fuzzy stochastic differential equations in M-type 2 Banach spaces. From now on we consider the fuzzy stochastic processes taking on values from $\mathcal{F L}_{c}^{b}(\mathcal{X})$, since for any fuzzy stochastic processes $x, y$ the measurability of the mapping $\omega \mapsto D_{\mathcal{X}}(x(t, \omega), y(t, \omega)), t \in I$, will be needed.

Proposition 5.18. Let $x: I \times \Omega \rightarrow \mathcal{F}_{c}^{b}(\mathcal{X})$ be a measurable and $L^{p}$-integrally bounded, $p \geq 1$. Then the fuzzy stochastic process $(t, \omega) \mapsto \int_{0}^{t} x(s, \omega) d s$ is measurable and takes on values from $\mathcal{F L}_{c}^{b}(\mathcal{X})$.

ProOF. By Remark 5.6 we get the measurability of $(t, \omega) \mapsto \int_{0}^{t} x(s, \omega) d s$. Also we have $\int_{0}^{t} x(s, \omega) d s \in \mathcal{F}_{c}^{b}(\mathcal{X})$. Let $\left\{\alpha_{k}\right\} \subset[0,1], \alpha \in(0,1]$ be such that $\alpha_{k} \nearrow \alpha$. Now for fixed $(t, \omega) \in I \times \Omega$ we get

$$
\begin{aligned}
H_{\mathcal{X}}\left(\left[\int_{0}^{t} x(s, \omega) d s\right]_{\alpha_{k}},\left[\int_{0}^{t} x(s, \omega) d s\right]_{\alpha}\right) & =H_{\mathcal{X}}\left(\int_{0}^{t}[x(s, \omega)]_{\alpha_{k}} d s, \int_{0}^{t}[x(s, \omega)]_{\alpha} d s\right) \\
& \leqslant \int_{0}^{t} H_{\mathcal{X}}\left([x(s, \omega)]_{\alpha_{k}},[x(s, \omega)]_{\alpha}\right) d s \\
& \leqslant \int_{0}^{T} H_{\mathcal{X}}\left([x(s, \omega)]_{\alpha_{k}},[x(s, \omega)]_{\alpha}\right) d s,
\end{aligned}
$$

and by the Lebesgue dominated convergence theorem $\int_{0}^{T} H_{\mathcal{X}}\left([x(s, \omega)]_{\alpha_{k}},[x(s, \omega)]_{\alpha}\right) d s \rightarrow 0$ as $k \rightarrow \infty$. This implies that $\int_{0}^{t} x(s, \omega) d s \in \mathcal{F}_{c}^{b}(\mathcal{X})$.

REMARK 5.19. Let $x: I \times \Omega \rightarrow \mathcal{F L}_{c}^{b}(\mathcal{X})$ be a nonanticipating and $L^{p}$-integrally bounded, $p \geq 1$. Then the fuzzy stochastic process $(t, \omega) \mapsto \int_{0}^{t} x(s, \omega) d s$ is nonanticipating and takes on values from $\mathcal{F}_{c}^{b}(\mathcal{X})$. 
Due to Remark 5.12 we can rewrite Proposition 5.8 with $\mathcal{F}_{c}^{b}(\mathcal{X})$ instead of $\mathcal{F}_{c}^{b}(\mathcal{X})$. Also the Propositions 5.9 and 5.10 are true if we replace $\mathcal{F}_{c}^{b}(\mathcal{X})$ with $\mathcal{F}_{c}^{b}(\mathcal{X})$. Such properties will be needed in the context of fuzzy stochastic differential equations considered in the next section.

Proposition 5.20. Assume that $x, y: I \times \Omega \rightarrow \mathcal{F L}_{c}^{b}(\mathcal{X})$ are measurable and $L^{p}$. integrally bounded, $p \geq 1$. Then $P$-a.e. it holds: for every $\tau<t$

$$
\sup _{u \in[\tau, t]} D_{\mathcal{X}}^{p}\left(\int_{\tau}^{u} x(s, \omega) d s, \int_{\tau}^{u} y(s, \omega) d s\right) \leqslant(t-\tau)^{p-1} \int_{\tau}^{t} D_{\mathcal{X}}^{p}(x(s, \omega), y(s, \omega)) d s
$$

and

$$
D_{\mathcal{X}}^{p}\left(\int_{\tau}^{t} x(s, \omega) d s, \int_{\tau}^{t} y(s, \omega) d s\right) \leqslant(t-\tau)^{p-1} \int_{\tau}^{t} D_{\mathcal{X}}^{p}(x(s, \omega), y(s, \omega)) d s .
$$

COROLlaRY 5.21. Under assumptions of Proposition 5.20, for every $\tau<t$ it holds

$$
\mathbb{E} \sup _{u \in[\tau, t]} D_{\mathcal{X}}^{p}\left(\int_{\tau}^{u} x(s) d s, \int_{\tau}^{u} y(s) d s\right) \leqslant(t-\tau)^{p-1} \mathbb{E} \int_{\tau}^{t} D_{\mathcal{X}}^{p}(x(s), y(s)) d s
$$

and

$$
\mathbb{E} D_{\mathcal{X}}^{p}\left(\int_{\tau}^{t} x(s) d s, \int_{\tau}^{t} y(s) d s\right) \leqslant(t-\tau)^{p-1} \mathbb{E} \int_{\tau}^{t} D_{\mathcal{X}}^{p}(x(s), y(s)) d s .
$$

6. Fuzzy stochastic differential equations in M-type 2 Banach spaces. Let $\mathcal{X}$ be a separable reflexive M-type 2 Banach space. We will consider fuzzy stochastic differential equations which are understood as the following integral equations

$$
x(t)=\phi(t) \oplus \int_{0}^{t} f(s, x(s)) d s \oplus\left\langle\left\langle\int_{0}^{t} g(s, x(s)) d W(s)\right\rangle, \quad t \in I,\right.
$$

which hold $P$-a.e. and where $\phi: I \times \Omega \rightarrow \mathcal{F L}_{c}^{b}(\mathcal{X}), f: I \times \Omega \times \mathcal{F}_{c}^{b}(\mathcal{X}) \rightarrow \mathcal{F}_{c}^{b}(\mathcal{X})$, $g: I \times \Omega \times \mathcal{F L}_{c}^{b}(\mathcal{X}) \rightarrow B(\mathcal{X} ; \mathcal{X})$. The first integral is the fuzzy stochastic Lebesgue integral and the second integral is the single-valued stochastic Itô integral in Banach space $\mathcal{X}$.

Such equations are some generalizations of the set-valued stochastic equations considered in Section 4. Here, we present the studies (parallel to those included in Section 4) on the equation (6.1). The derivations are very similar to those presented for the set-valed stochastic equation (4.1). For a clarity we include some sketches of the first proofs only.

Definition 6.1. A fuzzy stochastic process $x: I \times \Omega \rightarrow \mathcal{F}_{c}^{b}(\mathcal{X})$ is called the solution to (6.1) if it is $\left\{\mathcal{A}_{t}\right\}$-adapted, $D_{\mathcal{X}}$-continuous and satisfies (6.1). A solution $x: I \times$ $\Omega \rightarrow \mathcal{F L}_{c}^{b}(\mathcal{X})$ is unique if $P$-a.e. it holds: $D_{\mathcal{X}}(x(t), y(t))=0$ for every $t \in I$, where $y: I \times \Omega \rightarrow \mathcal{F L}_{c}^{b}(\mathcal{X})$ is any solution to (6.1). 
To obtain the existence and uniqueness theorem for solutions to (6.1) we will impose the following conditions:

(a1) $\phi: I \times \Omega \rightarrow \mathcal{F L}_{c}^{b}(\mathcal{X})$ is a nonanticipating, $D_{\mathcal{X}}$-continuous fuzzy stochastic process such that $\mathbb{E} \sup _{t \in I}\left|[\phi(t)]_{0}\right|_{\mathcal{X}}^{2}<\infty$,

(a2) $f: I \times \Omega \times \mathcal{F L}_{c}^{b}(\mathcal{X}) \rightarrow \mathcal{F L}_{c}^{b}(\mathcal{X})$ is $\mathcal{N} \otimes \beta_{D_{\mathcal{X}}^{S}} \mid \beta_{D_{\mathcal{X}}^{S}}$-measurable, $g: I \times \Omega \times$ $\mathcal{F L}_{c}^{b}(\mathcal{X}) \rightarrow B(\mathcal{X} ; \mathcal{X})$ is $\mathcal{N} \otimes \beta_{D_{\mathcal{X}}^{S}} \mid \sigma(B(\mathcal{X} ; \mathcal{X}))$-measurable,

(a3) there exists a constant $K>0$ such that $P$-a.e. it holds

$$
\begin{gathered}
\forall t \in I \quad \forall u, v \in \mathcal{F}_{c}^{b}(\mathcal{X}) \quad D_{\mathcal{X}}^{2}(f(t, \omega, u), f(t, \omega, v)) \leqslant K D_{\mathcal{X}}^{2}(u, v), \\
\forall t \in I \quad \forall u, v \in \mathcal{F}_{c}^{b}(\mathcal{X}) \quad\|g(t, \omega, u)-g(t, \omega, v)\|_{L^{2}(\mathcal{X} ; \mathcal{X})}^{2} \leqslant K D_{\mathcal{X}}^{2}(u, v),
\end{gathered}
$$

(a4) there exists a constant $M>0$ such that $P$-a.e. it holds

$$
\forall t \in I \quad \max \left\{\left|[f(t, \omega,\langle\langle\theta \mathcal{X}\rangle\rangle)]_{0}\right|_{\mathcal{X}}^{2},\|g(t, \omega,\langle\langle\theta \mathcal{X}\rangle\rangle)\|_{L^{2}(\mathcal{X} ; \mathcal{X})}^{2}\right\} \leqslant M .
$$

To use the method of the successive approximations we define the sequence $\left\{x_{n}\right\}_{n=0}^{\infty}$ of the fuzzy stochastic processes

$$
x_{0}(t)=\phi(t), \quad t \in I,
$$

and for $n=1,2, \ldots$

$$
x_{n}(t)=\phi(t) \oplus \int_{0}^{t} f\left(s, x_{n-1}(s)\right) d s \oplus\left\langle\left\langle\int_{0}^{t} g\left(s, x_{n-1}(s)\right) d W(s)\right\rangle, \quad t \in I .\right.
$$

LEMMA 6.2. Assume that $\phi, f, g$ satisfy conditions (al)-(a4). Then every approximation $x_{n}: I \times \Omega \rightarrow \mathcal{F L}_{c}^{b}(\mathcal{X})$ is a nonanticipating, $L^{2}$-integrally bounded, $D_{\mathcal{X}}$-continuous fuzzy stochastic process.

ProOF. By the assumptions on $\phi$, the initial approximation $x_{0}: I \times \Omega \rightarrow \mathcal{F L}_{c}^{b}(\mathcal{X})$ is such as it is stated in this lemma. Assume that $x_{n-1}$ is nonanticipating, $L^{2}$-integrally bounded and $D_{\mathcal{X}}$-continuous.

The compositions $f\left(\cdot, \cdot, x_{n-1}(\cdot, \cdot)\right): I \times \Omega \rightarrow \mathcal{F}_{c}^{b}(\mathcal{X}), g\left(\cdot, \cdot, x_{n-1}(\cdot, \cdot)\right): I \times \Omega \rightarrow$ $B(\mathcal{X} ; \mathcal{X})$ are $\mathcal{N} \mid \beta_{D_{\mathcal{X}}^{S}}$-measurable and $\mathcal{N} \mid \beta(B(\mathcal{X} ; \mathcal{X}))$-measurable, respectively. Next by (a3) and (a4) we obtain

$$
\begin{aligned}
\mathbb{E} \int_{I}\left|\left[f\left(t, x_{n-1}(t)\right)\right]_{0}\right|_{\mathcal{X}}^{2} d t & =\mathbb{E} \int_{I} D_{\mathcal{X}}^{2}\left(f\left(t, x_{n-1}(t)\right),\langle\langle\theta \mathcal{X}\rangle\rangle\right) d t \\
& \leqslant 2 K \mathbb{E} \int_{I}\left|\left[x_{n-1}(t)\right]_{0}\right|_{\mathcal{X}}^{2} d t+2 M T<\infty
\end{aligned}
$$

Also

$$
\mathbb{E} \int_{I}\left\|g\left(t, x_{n-1}(t)\right)\right\|_{L^{2}(\mathcal{X} ; \mathcal{X})}^{2} d t \leqslant 2 K \mathbb{E} \int_{I}\left|\left[x_{n-1}(t)\right]_{0}\right|_{\mathcal{X}}^{2} d t+2 M T<\infty .
$$


Therefore the first integral which appears in (6.3) is a nonanticipating and $L^{2}$-integrally bounded fuzzy stochastic process and the second integral is a well-defined $\mathcal{X}$-valued Itô stochastic integral. Hence $x_{n}$ is nonanticipating and $L^{2}$-integrally bounded. Since the integrals are continuous we conclude that every $x_{n}$ is $D_{\mathcal{X}}$-continuous.

LEMMA 6.3. Under assumptions of Lemma 6.2, for every $n \in \mathbb{N}$ it holds

$$
\mathbb{E} \sup _{t \in I} D_{\mathcal{X}}^{2}\left(x_{n}(t),\langle\langle\theta \mathcal{X}\rangle\rangle\right) \leqslant\left(M_{1}+M_{2} T \mathbb{E} \sup _{t \in I}\left|[\phi(t)]_{0}\right|_{\mathcal{X}}^{2}\right) \exp \left\{M_{2} T\right\}
$$

where $M_{1}=3 \mathbb{E} \sup _{t \in I}\left|[\phi(t)]_{0}\right|_{\mathcal{X}}^{2}+6 T M\left(T+C_{\mathcal{X}}\right), M_{2}=6 K\left(T+C_{\mathcal{X}}\right)$.

Proof. Due to Corollary 5.21, (2.2), (a3) and (a4) we have for $t \in I$

$$
\mathbb{E} \sup _{s \in[0, t]} D_{\mathcal{X}}^{2}\left(x_{n}(s),\langle\langle\theta \mathcal{X}\rangle\rangle\right) \leqslant M_{1}+M_{2} \int_{0}^{t} \mathbb{E} \sup _{s \in[0, r]} D_{\mathcal{X}}^{2}\left(x_{n-1}(s),\langle\langle\theta \mathcal{X}\rangle\rangle\right) d r .
$$

Hence, for every $k \in \mathbb{N}$ and every $t \in I$ we can infer that

$$
\begin{aligned}
\max _{1 \leqslant n \leqslant k} \mathbb{E} \sup _{s \in[0, t]} D_{\mathcal{X}}^{2}\left(x_{n}(s),\langle\langle\theta \mathcal{X}\rangle\rangle\right) \leqslant & M_{1}+M_{2} T \mathbb{E} \sup _{s \in I}\left|[\phi(s)]_{0}\right|_{\mathcal{X}}^{2} \\
& +M_{2} \int_{0}^{t} \max _{1 \leqslant n \leqslant k} \mathbb{E} \sup _{s \in[0, r]} D_{\mathcal{X}}^{2}\left(x_{n}(s),\langle\langle\theta \mathcal{X}\rangle\rangle\right) d r .
\end{aligned}
$$

Using Gronwall's inequality we obtain

$$
\max _{1 \leqslant n \leqslant k} \mathbb{E} \sup _{s \in[0, t]} D_{\mathcal{X}}^{2}\left(x_{n}(s),\langle\langle\theta \mathcal{X}\rangle\rangle\right) \leqslant\left(M_{1}+M_{2} T \mathbb{E} \sup _{s \in I}\left|[\phi(s)]_{0}\right|_{\mathcal{X}}^{2}\right) e^{M_{2} t}
$$

for $t \in I$, which leads us to the end of the proof.

THEOREM 6.4. Assume that $\phi, f, g$ satisfy (al)-(a4). Then the fuzzy stochastic differential equation (6.1) has a unique solution.

Proof. By Corollary 5.21, (2.2), (a3) and (a4) we have (for $t \in I$ )

$$
\mathbb{E} \sup _{s \in[0, t]} D_{\mathcal{X}}^{2}\left(x_{1}(s), x_{0}(s)\right) \leqslant L t
$$

where $L=4\left(T+C_{\mathcal{X}}\right)\left(M+K \mathbb{E} \sup _{t \in I}\left|[\phi(t)]_{0}\right|_{\mathcal{X}}^{2}\right)$. Further, for $n \in \mathbb{N}$ and $t \in I$ we obtain

$$
\mathbb{E} \sup _{s \in[0, t]} D_{\mathcal{X}}^{2}\left(x_{n+1}(s), x_{n}(s)\right) \leqslant 2 K\left(T+C_{\mathcal{X}}\right) \int_{0}^{t} \mathbb{E} \sup _{s \in[0, r]} D_{\mathcal{X}}^{2}\left(x_{n}(s), x_{n-1}(s)\right) d r .
$$

Therefore

$$
\mathbb{E} \sup _{t \in I} D_{\mathcal{X}}^{2}\left(x_{n}(t), x_{n-1}(t)\right) \leqslant L\left[2 K\left(T+C_{\mathcal{X}}\right)\right]^{n-1} \frac{T^{n}}{n !} .
$$


Hence we can infer about the existence of a fuzzy stochastic process $x: I \times \Omega \rightarrow \mathcal{F L}_{c}^{b}(\mathcal{X})$ which is nonanticipating, $L_{\mathcal{N}}^{2}(\lambda \times P)$-integrally bounded, $D_{\mathcal{X}}$-continuous and $P$-a.e. it holds

$$
\sup _{t \in I} D_{\mathcal{X}}\left(x_{n}(t, \omega), x(t, \omega)\right) \rightarrow 0 \text { as } n \rightarrow \infty .
$$

By Corollary 5.21, (2.1) and (a3) we have for every $t \in I$

$$
\begin{aligned}
& \mathbb{E} D_{\mathcal{X}}^{2}\left(x(t), \phi(t) \oplus \int_{0}^{t} f(s, x(s)) d s \oplus\left\langle\left\langle\int_{0}^{t} g(s, x(s)) d W(s)\right\rangle\right)\right) \\
& \leqslant 2 \mathbb{E} D_{\mathcal{X}}^{2}\left(x(t), x_{n}(t)\right)+4\left(T+C_{\mathcal{X}}\right) K \int_{I} \mathbb{E} D_{\mathcal{X}}^{2}\left(x_{n-1}(s), x(s)\right) d s,
\end{aligned}
$$

and since the right-hand side of this inequality converges to zero as $n \rightarrow \infty$, we obtain for every fixed $t \in I$

$$
D_{\mathcal{X}}\left(x(t), \phi(t) \oplus \int_{0}^{t} f(s, x(s)) d s \oplus\left\langle\left\langle\int_{0}^{t} g(s, x(s)) d W(s)\right\rangle\right)\right)=0 \quad P \text {-a.e. }
$$

Since the processes appearing above are $D_{\mathcal{X}}$-continuous, we get that $P$-a.e. it holds

$$
x(t)=\phi(t) \oplus \int_{0}^{t} f(s, x(s)) d s \oplus\left\langle\left\langle\int_{0}^{t} g(s, x(s)) d W(s)\right\rangle\right\rangle \text { for } t \in I .
$$

Hence $x$ is a solution to (6.1). Suppose that $y: I \times \Omega \rightarrow \mathcal{F L}_{c}^{b}(\mathcal{X})$ is also a solution to (6.1). Then for every $t \in I$

$$
\mathbb{E} \sup _{s \in[0, t]} D_{\mathcal{X}}^{2}(x(s), y(s)) \leqslant 2 K\left(T+C_{\mathcal{X}}\right) \int_{0}^{t} \mathbb{E} \sup _{s \in[0, r]} D_{\mathcal{X}}^{2}(x(s), y(s)) d r .
$$

This allows us to infer that $P$-a.e. it holds $\sup _{s \in I} D \mathcal{X}(x(s), y(s))=0$ which means that $x$ is the unique solution to (6.1).

PROPOSITION 6.5. Under assumptions of Theorem 6.4 for the solution $x$ to (6.1) and the $n^{\text {th }}$ approximation $x_{n}$ it holds

$$
\mathbb{E} \sup _{t \in I} D_{\mathcal{X}}^{2}\left(x_{n}(t), x(t)\right) \leqslant 2 L T \exp \left\{4 K T\left(T+C_{\mathcal{X}}\right)\right\} \frac{\left[2 K T\left(T+C_{\mathcal{X}}\right)\right]^{n}}{n !},
$$

where $L=4\left(T+C_{\mathcal{X}}\right)\left(M+K \mathbb{E} \sup _{t \in I}\left|[\phi(t)]_{0}\right|_{\mathcal{X}}^{2}\right)$. In particular,

$$
\mathbb{E} \sup _{t \in I} D_{\mathcal{X}}^{2}\left(x_{n}(t), x(t)\right) \rightarrow 0 \text { as } n \rightarrow \infty .
$$

Let us consider the equation (6.1) and the equation

$$
\tilde{x}(t)=\tilde{\phi}(t) \oplus \int_{0}^{t} \tilde{f}(s, \tilde{x}(s)) d s \oplus\left\langle\left\langle\int_{0}^{t} \tilde{g}(s, \tilde{x}(s)) d W(s)\right\rangle\right\rangle, \quad t \in I .
$$


Proposition 6.6. Assume that $\phi, \tilde{\phi}, f, \tilde{f}, g, \tilde{g}$ satisfy (al)-(a4). Then for the solution $x$ to (6.1) and the solution $\tilde{x}$ to (6.5) it holds

$$
\begin{aligned}
& \mathbb{E} \sup _{t \in I} D_{\mathcal{X}}^{2}(\tilde{x}(t), x(t)) \\
& \leqslant {\left[3 \mathbb{E} \sup _{t \in I} D_{\mathcal{X}}^{2}(\tilde{\phi}(t), \phi(t))+6 T \mathbb{E} \int_{I} D_{\mathcal{X}}^{2}(\tilde{f}(s, x(s)), f(s, x(s))) d s\right.} \\
&\left.+6 C_{\mathcal{X}} \mathbb{E} \int_{I}\|\tilde{g}(s, x(s))-g(s, x(s))\|_{L^{2}(\mathcal{X} ; \mathcal{X})}^{2} d s\right] \exp \left\{6 K T\left(T+C_{\mathcal{X}}\right)\right\} .
\end{aligned}
$$

Now let us consider the equation (6.1) and the equations

$$
y_{n}(t)=\phi_{n}(t) \oplus \int_{0}^{t} f_{n}\left(s, y_{n}(s)\right) d s \oplus\left\langle\left\langle\int_{0}^{t} g_{n}\left(s, y_{n}(s)\right) d W(s)\right\rangle\right\rangle, \quad t \in I,
$$

$n \in \mathbb{N}$.

COROLlary 6.7. Assume that $\phi, \phi_{n}, f, f_{n}, g, g_{n}$ satisfy (al)-(a4). Suppose that

$$
\mathbb{E} \sup _{t \in I} D_{\mathcal{X}}^{2}\left(\phi_{n}(t), \phi(t)\right) \rightarrow 0 \text { as } n \rightarrow \infty,
$$

and for every $u \in \mathcal{F L}_{c}^{b}(\mathcal{X})$

$$
\begin{gathered}
\mathbb{E} \int_{I} D_{\mathcal{X}}^{2}\left(f_{n}(s, u), f(s, u)\right) \rightarrow 0 \text { as } n \rightarrow \infty, \\
\mathbb{E} \int_{I}\left\|g_{n}(s, u)-g(s, u)\right\|_{L^{2}(\mathcal{X} ; \mathcal{X})}^{2} \rightarrow 0 \text { as } n \rightarrow \infty .
\end{gathered}
$$

Then for the solution $x$ to (6.1) and the solutions $y_{n}$ to (6.6) it holds

$$
\mathbb{E} \sup _{t \in I} D_{\mathcal{X}}^{2}\left(y_{n}(t), x(t)\right) \rightarrow 0 \text { as } n \rightarrow \infty .
$$

Acknowledgements. The author would like to express his gratitude for the referees' comments that improved the paper.

\section{REFERENCES}

[ 1] R. Atar, A. Budhiraja And K. Ramanan, Deterministic and stochastic differential inclusions with multiple surfaces of discontinuity, Probab. Theory Related Fields 142 (2008), 249-283.

[2] N. U. Ahmed, Nonlinear stochastic differential inclusions on Banach space, Stochastic Anal. Appl. 12 (1994), $1-10$.

[ 3 ] J.-P. Aubin And G. DA Prato, The viability theorem for stochastic differential inclusions, Stochastic Anal. Appl. 16 (1998), 1-15.

[4] J.-P. Aubin, G. Da Prato And H. Frankowska, Stochastic invariance for differential inclusions, SetValued Anal. 8 (2000), 181-201.

[ 5 ] J.-P. Aubin And H. Frankowska, Set-Valued Analysis, Birkhäuser, Basel, 1990. 
[6] P. Balasubramaniam and S. K. NTOuYas, Controllability for neutral stochastic functional differential inclusions with infinite delay in abstract space, J. Math. Anal. Appl. 324 (2006), 161-176.

[ 7 ] T. F. BRidgland (JR.), Trajectory integrals of set valued functions, Pacific J. Math. 33 (1970), $43-68$.

[ 8 ] Z. BRZEŹNIAK, Stochastic partial differential equations in M-type 2 Banach spaces, Potential Anal. 4 (1995), $1-45$.

[9] Z. BrzeźniaK And A. Carroll, Approximations of the Wong-Zakai differential equations in M-type 2 Banach spaces with applications to loop spaces, Séminaire de Probabilitiés 37, 251-289, Lecture Notes in Math., 1832, Springer, Berlin, 2003.

[10] Z. Brzeźniak And K. D. Elworthy, Stochastic differential equations on Banach manifolds, Methods Funct. Anal. Topology 6 (2000), 43-84.

[11] C. Castaing And M. Valadier, Convex Analysis and Measurable Multifunctions, Lecture Notes in Mathematics 580, Springer-Verlag, Berlin-New York, 1977.

[12] E. CÉPA, Équations différentielles stochastiques multivoques, Séminaire de Probabilités, XXIX, 86-107, Lecture Notes in Math. 1613, Springer, Berlin, 1995.

[13] A. Colubi, J. S. Domínguez-Menchero, M. López-Díaz and D. A. Ralescu, A $D_{E}[0,1]$ representation of random upper semicontinuous functions, Proc. Amer. Math. Soc. 130 (2002), 3237-3242.

[14] G. Da Prato and J. ZabCZyK, Stochastic Equations in Infinite Dimensions, Encyclopedia Math. Appl. 44, Cambridge University Press, Cambridge, 1992.

[15] J. L. Doob, Stochastic Processes, John Wiley \& Sons, New York, 1990.

[16] D. Dubois And H. Prade, Fuzzy sets and systems. Theory and applications. With a foreword by Lotfi A. Zadeh, Math. Sci. Engrg. 144, Academic Press, Inc., New York-London, 1980.

[17] N. Dunford And J. T. Schwartz, Linear Operators. Part I. General Theory. With the assistance of William G. Bade and Robert G. Bartle. Reprint of the 1958 original, Wiley Classics Lib. A Wiley-Interscience Publication. John Wiley \& Sons, Inc., New York, 1988.

[18] I. I. Gihman And A. V. Skorohod, Stochastic Differential Equations, Springer-Verlag, Berlin, 1972.

[19] D. GRIFFEATH, Limit theorems for nonergodic set-valued Markov processes, Ann. Probability 6 (1978), 379387.

[20] T. E. HARris, Additive set-valued Markov processes and graphical methods, Ann. Probability 6 (1978), 355378.

[21] F. HIAI, Convergence of conditional expectations and strong laws of large numbers for multivalued variables, Trans. Amer. Math. Soc. 120 (1985), 369-389.

[22] F. HiAI AND H. UMEGAKI, Integrals, conditional expectation, and martingales of multivalued functions, J. Multivariate Anal. 7 (1977), 149-182.

[23] S. Hu And N. Papageorgiou, Handbook of Multivalued Analysis. Vol. I. Theory, Math. Appl. 419, Kluwer Academic Publishers, Boston, 1997.

[24] N. IKeda and S. Watanabe, Stochastic Differential Equations and Diffusion Processes, North-Holland Math. Library 24, North-Holland Publishing Co., Amsterdam-New York; Kodansha, Ltd., Tokyo, 1981.

[25] M. KisielewiCZ, Properties of solution set of stochastic inclusions, J. Appl. Math. Stochastic Anal. 6 (1993), 217-235.

[26] E. P. Klement, M. L. Puri And D. A. Ralescu, Limit theorems for fuzzy random variables, Proc. Roy. Soc. London Ser. A 407 (1986), 171-182.

[27] P. KRÉE, Diffusion equation for multivalued stochastic differential equations, J. Funct. Anal. 49 (1982), $73-$ 90.

[28] K. Kuratowski, Topology, Vol. I, Adademic Press, New York, 1966.

[29] C. C. A. LABUSCHAGNE, A Banach lattice approach to convergent integrably bounded set-valued martingales and their positive parts, J. Math. Anal. Appl. 342 (2008), 780-797.

[30] V. Lakshmikantham and R. N. Mohapatra, Theory of Fuzzy Differential Equations and Inclusions, Ser. Math. Anal. Appl. 6, Taylor \& Francis, London, 2003. 
[31] D. LÉPIngle And C. Marois, Equations differentielles stochastiques multivoques unidimensionnelles, Séminaire de Probabilités, XXI, 520-533, Lecture Notes in Math. 1247, Springer, Berlin, 1987.

[32] J. G. LI, S. Li AND Y. OGURA, Strong solution of Itô type set-valued stochastic differential equation, Acta Math. Sin. (Engl. Ser.) 26 (2010), 1739-1748.

[33] S. Li AND Y. OGURA, Convergence of set valued sub- and supermartingales in the Kuratowski-Mosco sense, Ann. Probability 26 (1998), 1384-1402.

[34] M. T. Malinowski, On random fuzzy differential equations, Fuzzy Sets and Systems 160 (2009), 31523165 .

[35] M. T. Malinowski, Random fuzzy differential equations under generalized Lipschitz condition, Nonlinear Anal. Real World Appl. 13 (2012), 860-881.

[36] M. T. Malinowski, Strong solutions to stochastic fuzzy differential equations of Itô type, Math. Comput. Modelling 55 (2012), 918-928.

[37] M. T. MalinowsKi, Itô type stochastic fuzzy differential equations with delay, Systems Control Lett. 61 (2012), 692-701.

[38] M. T. Malinows Ki, Some properties of strong solutions to stochastic fuzzy differential equations, Inform. Sci. 252 (2013), 62-80.

[39] M. T. Malinows Ki, Approximation schemes for fuzzy stochastic integral equations, Appl. Math. Comput. 219 (2013), 11278-11290.

[40] M. T. MALINOWSKI, On a new set-valued integral with respect to semimartingales and its applications, J. Math. Anal. Appl. 408 (2013), 669-680.

[41] I. Mitoma, Y. OKAZAKi AND J. Zhang, Set-valued stochastic differential equation in M-type 2 Banach space, Comm. Stoch. Anal. 4 (2010), 215-237.

[42] I. Molchanov, Theory of Random Sets, Springer-Verlag, London, 2005.

[43] C. V. Negoita And D. A. Ralescu, Representation theorems for fuzzy concepts, Kybernetes 4 (1975), 169-174.

[44] C. V. Negoita And D. A. Ralescu, Applications of Fuzzy Sets to System Analysis, Wiley, New York, 1975.

[45] Y. Ogura And T. SETokUChi, Large deviations for random upper semicontinuous functions, Tohoku Math. J. 61 (2009), 213-223.

[46] B. ØKSENDAL, Stochastic Differential Equations. An Introduction with Applications, Sixth edition, Universitext, Springer Verlag, Berlin, 2003.

[47] M. L. PuRi AND D. A. RAlescu, Strong law of large numbers for Banach space valued random sets, Ann. Probability 11 (1983), 222-224.

[48] M. L. PuRI AND D. A. Ralescu, The concept of normality for fuzzy random variables, Ann. Probability 13 (1985), 1373-1379.

[49] M. L. Puri And D. A. Ralescu, Fuzzy random variables, J. Math. Anal. Appl. 114 (1986), 409-422.

[50] H. RÅDSTRÖM, An embedding theorem for spaces of convex sets, Proc. Amer. Math. Soc. 3 (1952), $165-169$.

[51] D. W. Stroock and S. R. Srinivasa Varadhan, Multidimensional Diffusion Processes, Grundlehren Math. Wiss. 233, Springer-Verlag, New York, 1979.

[52] J. M. A. M. Van Neerven, M. C. Veraar and L. Weis, Stochastic integration in UMD Banach spaces, Ann. Probability 35 (2007), 1438-1478.

[53] L. A. ZADEH, Fuzzy sets, Information and Control 8 (1965), 338-353.

[54] J. Zhang, S. Li, I. Mitoma AND Y. OKAZAKI, On the solutions of set-valued stochastic differential equations in M-type 2 Banach spaces, Tohoku Math. J. 61 (2009), 417-440.

[55] X. ZHANG, Skorohod problem and multivalued stochastic evolution equations in Banach spaces, Bull. Sci. Math. 131 (2007), 175-217.

[56] H.-J. Zimmermann, Fuzzy Set Theory-and its Applications, With a foreword by L. A. Zadeh, Second edition, Kluwer Academic Publishers, Boston, MA, 1992. 
INSTITUTE OF MATHEMATICS

CRACOW UNIVERSITY OF TECHNOLOGY

UL. WARSZAWSKA 24

31-155 KRAKÓW

POLAND

E-mail addresses: malinowskimarek@poczta.fm 